\title{
Forecasting with Alternative Data
}

\author{
MICHAEL FLEDER, Massachusetts Institute of Technology, USA \\ DEVAVRAT SHAH, Massachusetts Institute of Technology, USA
}

We consider the problem of forecasting fine-grained company financials, such as daily revenue, from two input types: noisy proxy signals a la alternative data (e.g. credit card transactions) and sparse ground-truth observations (e.g. quarterly earnings reports). We utilize a classical linear systems model to capture both the evolution of the hidden or latent state (e.g. daily revenue), as well as the proxy signal (e.g. credit cards transactions). The linear system model is particularly well suited here as data is extremely sparse (4 quarterly reports per year). In classical system identification, where the central theme is to learn parameters for such linear systems, unbiased and consistent estimation of parameters is not feasible: the likelihood is non-convex; and worse, the global optimum for maximum likelihood estimation is often non-unique.

As the main contribution of this work, we provide a simple, consistent estimator of all parameters for the linear system model of interest; in addition the estimation is unbiased for some of the parameters. In effect, the additional sparse observations of aggregate hidden state (e.g. quarterly reports) enable system identification in our setup that is not feasible in general. For estimating and forecasting hidden state (actual earnings) using the noisy observations (daily credit card transactions), we utilize the learned linear model along with a natural adaptation of classical Kalman filtering (or Belief Propagation). This leads to optimal inference with respect to mean-squared error. Analytically, we argue that even though the underlying linear system may be "unstable," "uncontrollable," or "undetectable" in the classical setting, our setup and inference algorithm allow for estimation of hidden state with bounded error. Further, the estimation error of the algorithm monotonically decreases as the frequency of the sparse observations increases. This, seemingly intuitive insight contradicts the word on the Street, cf. [29]. Finally, we utilize our framework to estimate quarterly earnings of 34 public companies using credit card transaction data. Our data-driven method convincingly outperforms the Wall Street consensus (analyst) estimates even though our method uses only credit card data as input, while the Wall Street consensus is based on various data sources including experts' input.

Keywords: Forecasting; Alternative Data; Linear Systems; Time Series; Finance; Consumer Credit Card Transactions

\section{ACM Reference Format:}

Michael Fleder and Devavrat Shah. 2019. Forecasting with Alternative Data. In Proc. ACM Meas. Anal. Comput. Syst., Vol. 3, 3, Article 46 (December 2019). ACM, New York, NY. 29 pages. https://doi.org/10.1145/3366694

\section{INTRODUCTION}

In recent years there has been a proliferation of alternative financial datasets ("alt data") that function as side-channel, or proxy information for company financials [30] [8]. For example, alt data sets consisting of consumer credit card transactions can be used to estimate daily consumer spending on Uber and Lyft [9] or at McDonald's [20]. Ground-truth for these numbers is almost never disclosed on a daily basis, but aggregates (e.g. total revenue) are reported at lower frequency

Authors' addresses: Michael Fleder, Massachusetts Institute of Technology, 32-D560 Vassar St. Cambridge, MA, 02139, USA, mfleder@mit.edu; Devavrat Shah, Massachusetts Institute of Technology, 32-D670 Vassar St. Cambridge, MA, 02139, USA, devavrat@mit.edu.

Permission to make digital or hard copies of all or part of this work for personal or classroom use is granted without fee provided that copies are not made or distributed for profit or commercial advantage and that copies bear this notice and the full citation on the first page. Copyrights for components of this work owned by others than the author(s) must be honored Abstracting with credit is permitted. To copy otherwise, or republish, to post on servers or to redistribute to lists, requires prior specific permission and/or a fee. Request permissions from permissions@acm.org.

(c) 2019 Copyright held by the owner/author(s). Publication rights licensed to ACM.

2476-1249/2019/12-ART46 \$15.00

https://doi.org/10.1145/3366694

Proc. ACM Meas. Anal. Comput. Syst., Vol. 3, No. 3, Article 46. Publication date: December 2019. 


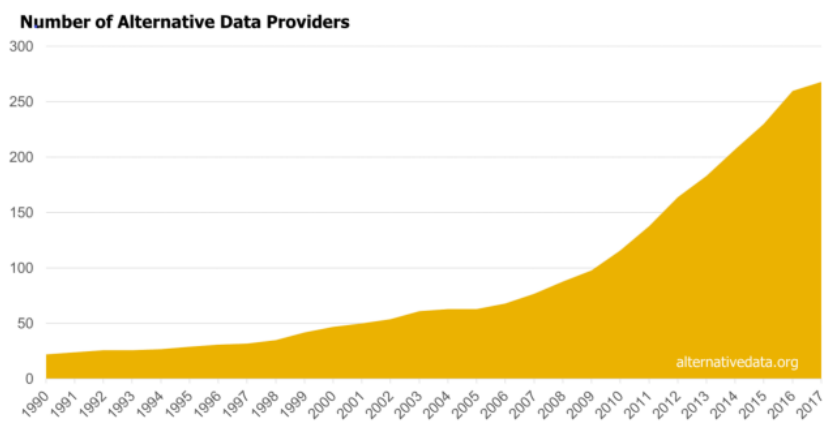

Fig. 1. Alternative data providers by year [2]

typically quarterly. Interest in alt data has grown significantly (See Figure 1, [31]) primarily because of alt data's promise to help estimate company financials at higher frequency than quarterly reports. However, combining alt data with lower-frequency ground truth (e.g. quarterly reports), for accurate, frequent estimation and forecasting of hidden company financials, remains challenging; and approaches are often highly dataset specific, cf. [1] and [6].

The primary goal of this work is to develop a method for accurately forecasting company financials by combining noisy, high-frequency alt data and lower-frequency aggregates (like quarterly reports). In addition, we require our forecasts to update frequently - with every new data point. The flip side of this forecasting exercise is to understand the implications of requiring companies to disclose ground truth in quarterly reports. We investigate how much is really revealed about higher-frequency dynamics through quarterly reports.

Contributions. We make three contributions. First, the main contribution of this work is providing a systematic approach for tracking company financials at high-frequency, where we combine lowfrequency ground-truth aggregates with high-frequency, noisy proxy data. We utilize a variation of the classical linear dynamical systems (LDS) model with hidden state and noisy observations with Gaussian noise. Compared to the classical setting, for example that considered in [5], we are also given infrequent observations of aggregate hidden state. Our goal is the same: to develop an estimation algorithm for the hidden state. We show that our model is effective with sparse observations - for example receiving alternative data weekly but aggregate observations every 3 months.

Our second contribution is solving the two problems required for utilizing this LDS model. First, we solve the problem of learning the model parameters, referred to as "system identification" in the classical literature. We show that the inclusion of infrequent aggregate state observations allows us to devise consistent estimators for all parameters, and provide a finite sample analysis of the resulting error. And for some of the parameters, the estimation is unbiased. This is surprising, because in the classical setup, system identification for LDS suffers from non-uniqueness: multiple sets of parameters give rise to identical likelihood values ([14] page 387); and furthermore, the likelihood is non-convex, making optimization challenging (see Figure 3). In contrast, our algorithms are consistent and computationally efficient: given $k$ observations of the latent state, a latent state of dimension $n$, and observations of dimension $m$, the running time of our system identification algorithms depends only on multiplication of matrices of size $(k \times n),(k \times m)$ and inversion of $n \times n$ matrices, for which there are efficient methods.

The next part of this contribution, of utilizing a LDS in a sparse data setting, is providing and analyzing an optimal inference algorithm. In the classical setting, Kalman Filtering, provides the 
optimal estimation procedure in terms of minimizing mean squared error. In our modified setup, this no longer holds. We develop such a method and provide an optimal estimation algorithm. We show that if ground-truth aggregate information is available with any non-zero expected frequency, then tracking estimation error remains bounded - even if the dynamics are unstable, uncontrollable, or undetectable. Furthermore, we show that the tracking estimation error decreases monotonically as ground-truth information becomes increasingly available - which is intuitively pleasing. This directly contradicts the claim General Motors Co. (GM) made in April 2018, when GM switched from monthly to quarterly reporting for its U.S. vehicle sales; GM stated that monthly sales are not useful for investors [29]. Per our analysis, having monthly versus quarterly earnings reports improves estimation of company financials; thus GM's claim is arguably incorrect.

Our third contribution is putting our model into practice. We start by empirically validating the theorems using synthetic data. Next, we apply our end-to-end identification and tracking algorithms to an alternative data set of real credit card transactions obtained from a hedge fund. The data set consists of typically weekly or biweekly summaries of unknown fractions of consumer spending at 34 public companies. The prediction task is to forecast weekly revenue (and hence quarterly earnings) at each of the companies using both the credit card data along with historical, public, quarterly disclosures of revenue. Our method outperforms a standard benchmark of Wall Street consensus estimates, beating the consensus on $57.2 \%$ of quarterly predictions as well as outperforming with respect to the mean-squared error. The model performance is significant, because we do not make use of any additional information or expert input that may have been available as input for other financial analysts' estimates.

Related System Identification Work. System identification for linear dynamical systems is a classic topic. A rough taxonomy of approaches is shown in Figure 2. The problem suffers from non-uniqueness, where multiple sets of parameters may give rise to the same likelihood ([14] page 387), as well as non-convexity of the likelihood. Without constraints on the parameters, the standard LDS is unidentifiable [14]. In our work, we do not constrain the parameter space; instead we impose a simple aggregate constraint on the state-space that arises naturally in financial applications. And this sparse aggregate constraint allows us to identify the system.

In the classical setup, two heuristic approaches for system identification are expectation-maximization (EM) and subspace identification [24]. Both start by approximating the hidden state and then estimating the system parameters. As [18] notes, neither EM nor subspace identification provides theoretical guarantees; and both can result in poor performance.

Hazad et. al. [18] [17] side-step the standard system identification problem and avoid learning the underlying system parameters. Instead, they construct a forecasting algorithm for predicting the observation sequence, with their predictions converging to those of the optimal LDS estimator with a corresponding regret bound. Their approach assumes a stable system and various constraints on the parameters. Hazad et. al. do not attempt to estimate the hidden state, which is of critical importance to us. The hidden state, in our applications, contains the quarterly financial disclosure that we wish to estimate.

Within a restricted class of LDS, gradient descent has been used successfully for system identification [16]. Hardt et. al. assume a system with no process noise and a constrained transition matrix [16]. Under these constraints, [16] demonstrates that gradient descent converges in polynomial time to the maximum likelihood solution. However, financial time-series are generally noisy. And so we focus on the more general case of LDS with both process and observation noise.

A somewhat brute-force approach to system identification is multiple model adaptive estimation (MMAE) [15]. MMAE concurrently runs a set of Kalman filters, each with different internal system parameters. Each filter produces a different residual sequence. Hypothesis testing procedures can 
then be constructed to select the most likely system from the proposed set [15]. An obvious difficulty here is proposing the candidate parameters, since we assume the parameter space is unconstrained. Finally, Karami et. al. [21] provide an alternative method for estimating the maximum likelihood solution for system identification. They start by estimating the parameters of a closely related system, before trying to recover the original linear dynamical system parameters. Their estimators are not consistent, and since the maximum likelihood solution may be biased (see Figure 3), their resulting estimators may be biased.

Related Inference Work. Designing and analyzing inference algorithms for linear dynamical systems is also a classic topic. An extended overview of the work and its relation to optimality of linear control with quadratic cost can be found in the books by Bertsekas [5] (see Chapter 3) and Goodwin [11]. The summary of this work is as follows. The conditional expectation of the hidden state (which naturally minimizes the mean-squared error) can be calculated in an iterative manner using Kalman filtering, also known as belief propagation for Gaussian graphical models. The statistical properties of this optimal filtering algorithm are extensively studied by analyzing the so-called Riccati equations. Specifically, under the conditions known as "detectability" and "controllability" on the system dynamics, it has been argued that the mean-squared error in estimating the hidden state converges to a finite value. And such conditions are necessary.

In our work we look to derive the equivalent of Kalman filtering for our augmented linear dynamical systems setup (see Section 2) and the conditions under which the mean-squared error in the estimation of hidden state remains bounded.

The relevant prior works that have attempted to address such an extension falls in two categories. First, non-linear systems dynamics and associated non-linear Kalman filtering have been studied with Extended Kalman Filters [11], Unscented Kalman Filters [28], Particle Filters [12] and related. While these methods have been very successful empirically, little is known about their statistical performance. Second, we look to the history of linear dynamical systems with missing observations. A recent study is by Sinopoli et. al. [27]. They present a model for Kalman filtering with missing observations and then analyze the convergence using a modified version of the Riccati equation. Sinopoli. et al. show that there is a threshold on the fraction of observed data, below which the mean-squared estimation error for hidden state diverges while above which the mean-squared error converges. This, however, requires that the underlying system, as in the classical setup, satisfies appropriate conditions such as "detectability" and "controllability." While our work takes inspiration from that of [27], it requires a different algorithm and analysis. For example, as a consequence of our result, we show that mean-squared error in the hidden state remains bounded even if the underlying linear system is not "detectable."

Next, we take note of work on linear systems dynamics with constraints on the state-space. For example, infrequent observations in our setup can be viewed as constraints on the hidden statespace. Work by Simon and Chia [26] describes projecting an unconstrained Kalman filter solution onto the constraint surface. A useful reference is work by Gupta [13]. However, statistical analysis for these approaches is lacking; and none of these approaches incorporate partial observations or observation frequency analysis.

Finally, we mention recurrent neural networks (RNNs). Recent work by Che et. al. [7] describes using RNNs for forecasting with missing values. However, the data sets used in [7] are orders of magnitude larger than what we encounter with alt data. In ours and many related financial applications, the data sets are small and sparse, so we need a parsimonious model and cannot rely on highly-parameterized RNNs. Furthermore, RNN's do not provide statistical guarantees, which can be critical for ours and similar applications. 


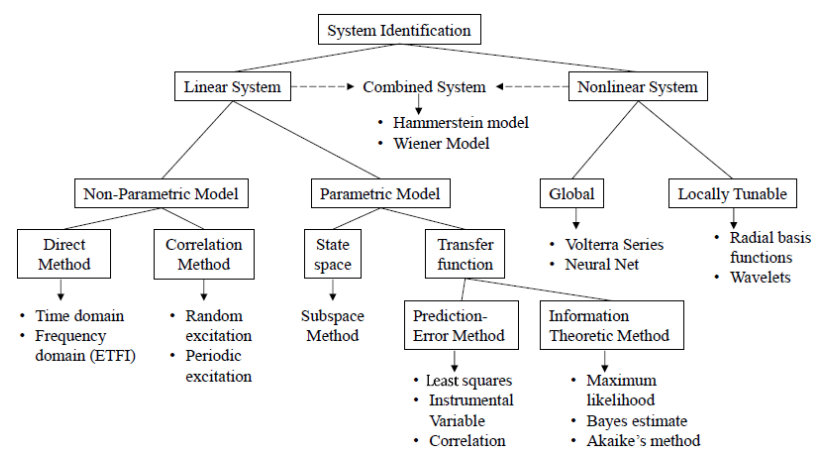

Fig. 2. Taxonomy of System Identification Approaches (taken from [4] [19]). Our work falls under Linear Systems / Parametric Model.

Outline. In Section 2. we formulate the alt data forecasting problem as a linear dynamical system with latent state, and partial and missing observations. We describe our modeling choices and then state the two main problems: (i) system identification and (ii) inference of the hidden state. In Section 3 we state the main theorems of the paper that (i) solve the system identification problem in this setting, and provide a finite sample analysis of the resulting error; (ii) state the optimal state estimator - which minimizes the mean-squared estimation error assuming the system has been identified; (iii) provide convergence conditions for the expected estimation error covariance and (iv) relate the observation frequency to the estimation error. We establish the quantitative and qualitative properties of estimation with respect to model parameters and data sparsity. Proofs for Section 3 are left to the Appendix. Section 4 presents experiments with both synthetic and real data. Synthetic data enables us to verify empirically the tightness of both the system identification and inference theorems. Then using real data, we argue that our method provides better quality estimation than Wall Street consensus estimates for the task of predicting key quarterly financials Our method outperforms this benchmark even though we do not make use of any other public information that Wall Street analysts might incorporate into their forecasts. We conclude in Section 5 .

\section{MODEL AND PROBLEM STATEMENT}

While our forecasting framework has a variety of applications, for ease of exposition we use the previously mentioned alt data problem as a running example. In particular, we examine forecasting daily company financials from two input sources (i) partial, noisy, daily observations of company financials (e.g. from some alt data source) and (ii) quarterly reports of ground-truth aggregates. This is a multiple-input, multiple-output (MIMO) system.

\subsection{Model}

We start by describing our model of financial data sources before justifying the modeling choices. Alternative Data Input: Let $x_{t} \in \mathbb{R}^{n}$ denote the hidden state vector of actual company financials on day $t \geq 0$. For simplicity, we ignore any non-negativity constraints on $x_{t}$. Let $y_{t} \in \mathbb{R}^{m}$ denote the alt data source - a linear, noisy observation of $x_{t}$ on day $t$. We assume $x_{t}$ follows an autoregressive process and that $x_{t}$ and $y_{t}$ are related via the following linear dynamical system equations: for $A \in \mathbb{R}^{n \times n}, C \in \mathbb{R}^{m \times n}$, and $t \in \mathbb{N}$,

$$
x_{t+1}=A x_{t}+w_{t} \quad y_{t}=C x_{t}+v_{t}
$$


where $w_{t} \in \mathbb{R}^{n}, v_{t} \in \mathbb{R}^{m}$ are independent and identically distributed random vectors with zero mean, covariance (positive definite) matrices $Q \in \mathbb{R}^{n \times n}, R \in \mathbb{R}^{m \times m}$ respectively. For simplicity of exposition, we shall assume that $Q, R$ are diagonal matrices, and $w_{t}, v_{t}$ have Gaussian distributions. Quarterly Reports Input: Let $s_{t} \in \mathbb{R}^{n}$ denote the running summation of the hidden state from day 0 to day $t \in \mathbb{N}$, as would be included in a quarterly report. We include the entire time period rather than just aggregation from a recent period as these two are equivalent and the cumulative representation is less cumbersome going forward. Let $y_{t}^{s} \in \mathbb{R}^{n}$ denote the observation of $s_{t}$ : for any $t \in \mathbb{N}$,

$$
s_{t}=\sum_{i=0}^{t} x_{i} \quad y_{t}^{s}=\left\{\begin{array}{ll}
s_{t}, & \gamma_{t}=1 \\
\text { missing, } & \gamma_{t}=0
\end{array}\right\}
$$

where $\left\{\gamma_{t}\right\}$ is a binary sequence determining the observation of $s_{t}$. For simplicity, we assume that the time between observations of $s_{t}$ is constant (e.g. quarterly); but we will relax this later. Figure 4 shows a graphical model for the problem.

Target: We want to track the latent $x_{t}$ and subsequently forecast future $s_{t}$.

Model fustification. The justification for our model is as follows. Many alternative datasets are available with limited history [3]. We might have at most a few fiscal quarters of data - possibly at weekly or bi-weekly frequency (see Section 4). That translates into just dozens of samples of alternative data, and a handful of corresponding quarterly reports. Thus, a linear model is a natural starting point for such a dynamical system. The number of key parameters $(A, C, Q, R)$ is minimal, so we might hope to infer both the parameters and latent variables from limited data.

Alternatively, our model could have incorporated: nonlinear state transitions, nonlinear observations, time-trends, and/or explicit periodic components. However, we see our application of linear dynamical systems here as a first step; and we hope that our work provides a framework within which more complex models can be developed.

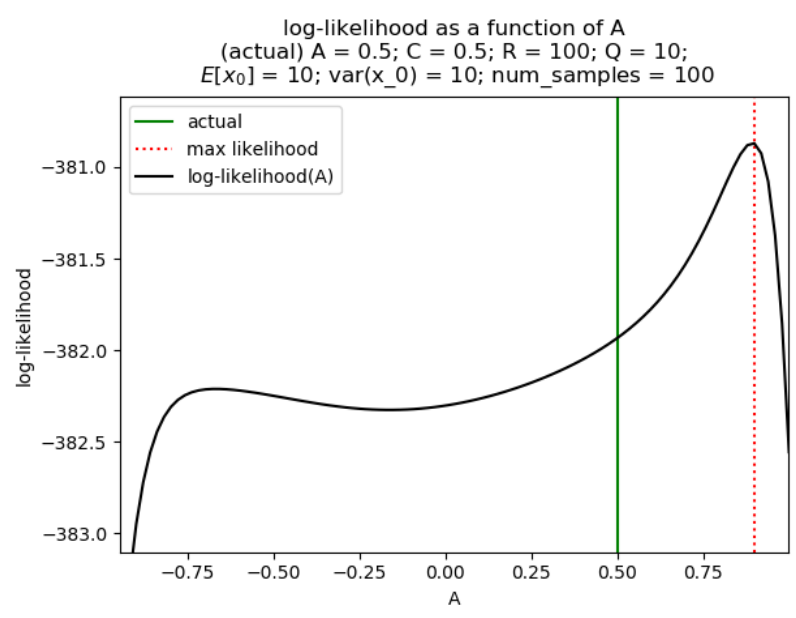

Fig. 3. Non-convex likelihood shown here as a function of $A$. The figure also indicates that the maximum likelihood solution may be biased. 


\subsection{System Identification}

Consider the previously described linear dynamical system with an intermittently observed, noisy, summation constraint: for $t \in \mathbb{N}$,

$$
\begin{aligned}
& x_{t+1}=A x_{t}+w_{t} \quad y_{t}=C x_{t}+v_{t} \quad s_{t}=\sum_{i=0}^{t} x_{i} \\
& \gamma_{t} \in\{0,1\} \quad y_{t}^{s}=\left\{\begin{array}{ll}
s_{t}, & \gamma_{t}=1 \\
\text { missing, } & \gamma_{t}=0
\end{array}\right\}
\end{aligned}
$$

We recall that

$$
\begin{array}{rlrl}
\mathbb{E}\left[w_{t} w_{t}^{\prime}\right]=Q=\operatorname{diag}\left(Q_{1,1}, \ldots, Q_{n, n}\right) & \mathbb{E}\left[w_{t}\right]=0 & A \neq 0 \\
\mathbb{E}\left[v_{t} v_{t}^{\prime}\right]=R=\operatorname{diag}\left(R_{1,1}, \ldots, R_{m, m}\right) & \mathbb{E}\left[v_{t}\right]=0 & C \neq 0
\end{array}
$$

In summary,

$$
x_{t}, w_{t}, s_{t} \in \mathbb{R}^{n}, y_{t}, v_{t} \in \mathbb{R}^{m}, C \in \mathbb{R}^{m \times n}, A \in \mathbb{R}^{n \times n}
$$

We assume that unique left inverse of $C$ exists, i.e. there exists $C^{-1} \in \mathbb{R}^{n \times m}$ so that $C^{-1} C=I \in \mathbb{R}^{n \times n}$. We assume that all the noise random variables $w_{i}, v_{j}$ are independent across all $i, j \geq 1$. Lastly, if $\left(t_{1}, \ldots, t_{k}\right)$ are the sequence of times for which $\gamma_{t}=1\left(s_{t_{i}}\right.$ observed), then for any $i$ s.t. $1 \leq i<$ $k,\left(t_{i+1}-t_{i}\right)$ is a constant independent of $i$ (e.g. observation every 90 days).

Goal: Determine $A, C, Q, R$.

\subsection{Inference}

We now state the inference problem. First define:

$$
\begin{gathered}
X_{t}:=\left(x_{t}, s_{t}\right) \quad Y_{t}:=\left(y_{t}, y_{t}^{s}\right) \quad Y_{\boldsymbol{t}}:=\left[Y_{0}, \ldots, Y_{t}\right]^{\prime} \\
\hat{X}_{t \mid t}:=\mathbb{E}\left[X_{t} \mid Y_{\boldsymbol{t}}, \gamma_{\boldsymbol{t}}\right] \quad \hat{X}_{t+1 \mid t}:=\mathbb{E}\left[X_{t+1} \mid Y_{\boldsymbol{t}}, \boldsymbol{\gamma}_{\boldsymbol{t}}\right] \\
P_{t \mid t}:=\mathbb{E}\left[\left(X_{t}-\hat{X}_{t \mid t}\right)\left(X_{t}-\hat{X}_{t \mid t}\right)^{\prime} \mid Y_{\boldsymbol{t}}, \boldsymbol{\gamma}_{\boldsymbol{t}}\right] \\
P_{t+1 \mid t}:=\mathbb{E}\left[\left(X_{t+1}-\hat{X}_{t+1 \mid t}\right)\left(X_{t+1}-\hat{X}_{t+1 \mid t}\right)^{\prime} \mid Y_{\boldsymbol{t}}, \gamma_{\boldsymbol{t}}\right]
\end{gathered}
$$

where $\gamma_{t}:=\left[\gamma_{0}, \ldots, \gamma_{t}\right]^{\prime}$.

Goal: For each $t$, we want to estimate both the hidden, augmented state vector $X_{t}$ along with the error covariance $P_{t \mid t}$.

\section{MAIN RESULTS}

\subsection{System Identification}

The system parameters to estimate are $A, C, Q$ and $R$. Table 1 and Table 2 summarize the properties and required inputs of our estimators. We start by defining the estimators before stating the main system identification theorems. Proofs are left to Section A.1. 


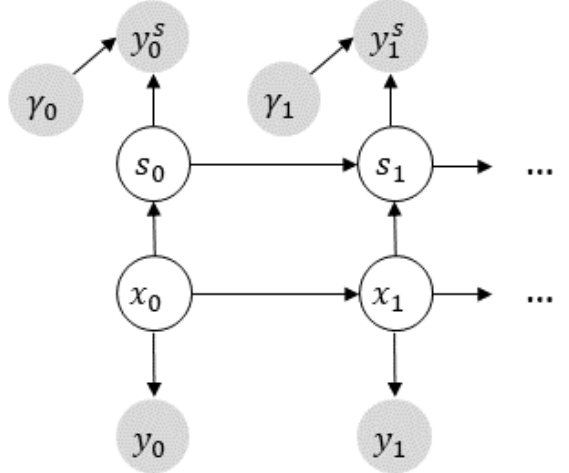

Fig. 4. Graphical model for the alt data tracking problem. The goal is to estimate $\left(x_{t}, s_{t}\right)$ at each time step. The $x_{i}$ are the latent daily company financials (e.g. revenue). The $y_{i}$ represent alt data - noisy observations of the $x_{i}$. And with some frequency $\lambda$ we receive a noiseless observation $y_{t}^{s}$ of $s_{t}$, an aggregation of all the $x_{i}$.

That is, $s_{t}=s_{t-1}+x_{t}$. The binary $\gamma_{t}$ determine whether $s_{t}$ is observed $\left(\gamma_{t}=1\right)$ or not $\left(\gamma_{t}=0\right)$.

\section{Estimator Unbiased Consistent Error Bound}

$\begin{array}{cccc}\hat{C} & \mathrm{Y} & \mathrm{Y} & \mathrm{Y} \\ \hat{R} & \mathrm{Y} & \mathrm{Y} & \mathrm{N} \\ \hat{A} & \mathrm{~N} & \mathrm{Y} & \mathrm{N} \\ \hat{Q} & \mathrm{~N} & \mathrm{Y} & \mathrm{N}\end{array}$

Table 1. Summary of estimator properties

Estimator

$$
\begin{array}{cc}
\hat{C} & \left\{\left(s_{t}, \Sigma_{0}^{t} y_{i}\right) \mid \gamma_{t}=1\right\} \\
\hat{R} & \left\{\left(s_{t}, \Sigma_{0}^{t} y_{i}\right) \mid \gamma_{t}=1\right\}, \hat{C} \\
\hat{A} & \left\{\left(s_{t}, y_{t}, y_{t+1}\right) \mid \gamma_{t}=1\right\}, y_{0}, \hat{C} \\
\hat{Q} & \left\{\left(s_{t}, y_{t}, y_{t+1}\right) \mid \gamma_{t}=1\right\}, y_{0}, \hat{C}, \hat{R}, \hat{A}
\end{array}
$$

Table 2. Summary of Estimator Arguments 
3.1.1 Definitions. Let $k \in \mathbb{Z}^{+}$be the number observations of the sum $s_{t}$ in a given training data set. Then let $\left(t_{1}, \ldots, t_{k}\right)$ be the sequence of $k$ times at which we observe $s_{t_{i}}$; so $\left\{s_{t_{i}}\right\}=\left\{s_{t} \mid \gamma_{t}=1\right\}$. And set $t_{0}:=-1$. Recall that $x_{t} \in \mathbb{R}^{n}$. Assume $k \geq n$. Define

$$
\begin{array}{cc}
S_{i}:=s_{t_{i}}-s_{t_{i-1}}=\sum_{j=t_{i-1}+1}^{t_{i}} x_{j} \quad & \tilde{S}_{i}:=\frac{S_{i}}{\sqrt{t_{i}-t_{i-1}}} \\
\tilde{Y}_{i}:=\frac{\sum_{t_{i-1}+1}^{t_{i}} y_{j}}{\sqrt{t_{i}-t_{i-1}}} & G_{i}^{\prime}(C):=\frac{C^{-1} y_{t_{i-1}+1}-S_{i}}{\sqrt{t_{i}-t_{i-1}-1}} \\
D_{i}^{\prime}(C):=\frac{\left(C^{-1} y_{t_{i}}-S_{i}\right)}{\sqrt{t_{i}-t_{i-1}-1}} \quad \tilde{Y}^{\prime}:=\left[\begin{array}{l}
-\tilde{Y}_{1}- \\
\vdots \\
-\tilde{Y}_{k}-
\end{array}\right] \\
\tilde{S}:=\left[\begin{array}{c}
-\tilde{S}_{1}- \\
\vdots \\
-\tilde{S}_{k}-
\end{array}\right] \\
D(C):=\left[\begin{array}{c}
-D_{1}(C)- \\
\vdots \\
-D_{k}(C)-
\end{array}\right] \quad G(C):=\left[\begin{array}{c}
-G_{1}(C)- \\
\vdots \\
-G_{k}(C)-
\end{array}\right]
\end{array}
$$

3.1.2 Estimator Definitions. Let $D=D(\hat{C}), G=G(\hat{C}), A=A(\hat{C})$. We shall assume $k \geq \max (m, n)$. Define

$$
\begin{gathered}
\hat{C}:=\left(\left(\tilde{S}^{\prime} \tilde{S}\right)^{-1} \tilde{S}^{\prime} \tilde{Y}\right)^{\prime} \\
\hat{R}(\hat{C}):=\frac{1}{k-n} \operatorname{diag}\left\{\left(\tilde{Y}-\tilde{S} \hat{C}^{\prime}\right)^{\prime}\left(\tilde{Y}-\tilde{S} \hat{C}^{\prime}\right)\right\} \\
\hat{A}(\hat{C}):=\left(\left(D^{\prime} D\right)^{-1} D^{\prime} G\right)^{\prime} \\
\hat{Q}(\hat{C}, \hat{R}, \hat{A}):=\frac{1}{k-n} \operatorname{diag}\left\{\left(G-D \hat{A}^{\prime}\right)^{\prime}\left(G-D \hat{A}^{\prime}\right)\right\} \\
\quad-\frac{1}{k} \sum_{i=1}^{k} \ell_{i}(\hat{C}, \hat{R}, \hat{A}) \\
\quad \ell_{i}(\hat{C}, \hat{R}, \hat{A}):=\frac{\hat{C}^{-1} \hat{R}\left(\hat{C}^{-1}\right)^{\prime}+\hat{A} \hat{C}^{-1} \hat{R}\left(\hat{A} \hat{C}^{-1}\right)^{\prime}}{t_{i}-t_{i-1}-1}
\end{gathered}
$$

\subsubsection{Formal Results.}

THEOREM $3.1(\hat{C})$. Recall that $k$ is the number observations of the sum $s_{t}$ in a given training data set; and $n, m$ are the dimensions of the latent state and observations respectively. Let $k \geq \max (m, n)$. Then

$$
\|C-\hat{C}\|_{F}^{2} \leq c\|R\|_{\infty, \infty} \frac{n+\log n+\log m+\log k}{k \lambda_{\min }\left(\frac{1}{k} \tilde{S}^{\prime} \tilde{S}\right)},
$$


with probability at least $1-1 /(m n k)$ for some universal constant $c>0$. Further, let the spectral radius of $A, \rho(A)$ be strictly less than 1 . Then

$$
\hat{C} \rightarrow C, \text { with probability } 1,
$$

as well as $\mathbb{E}[\hat{C}]=C$ for all $k \geq \max (m, n)$. Intuitively, the more frequent our observations of aggregate state, the tighter the error bound; and in the limit, the error tends to 0.

Theorem $3.2(\hat{R})$. Let the spectral radius of $A, \rho(A)$ be strictly less than 1 . Then,

$$
\mathbb{E}[\hat{R}]=R,
$$

and $\hat{R} \rightarrow R$ with probability 1 as $k \rightarrow \infty$.

Theorem $3.3(\hat{A})$. Let the spectral radius of $A, \rho(A)$ be strictly less than 1 . Then,

$$
\hat{A}(\hat{C}) \rightarrow A \text {, with probability } 1,
$$

as $k \rightarrow \infty$. That is, $\hat{A}(\hat{C})$ is consistent.

Theorem $3.4(\hat{Q})$. Let the spectral radius of $A, \rho(A)$ be strictly less than 1 . Then,

$$
\hat{Q}(\hat{C}, \hat{R}, \hat{A}) \rightarrow Q \text {, with probability } 1,
$$

as $k \rightarrow \infty$. That is, $\hat{Q}(\hat{C}, \hat{R}, \hat{A})$ is consistent.

\subsection{Inference}

We now assume the system has been identified. Our key inference results relate the frequency of observation for $s_{t}$ (determined by $\left\{\gamma_{t}\right\}$ ) to the expected error covariance. Whereas for system identification we assumed periodic observation of $s_{t}$, here we augment the model and let $\left\{\gamma_{t}\right\}$ follow a Bernoulli process with parameter $\lambda \in[0,1]$. Proofs are left to Section A.2. For shorthand, we let $P_{t}:=P_{t \mid t-1}$.

Theorem 3.5 (Phase Transition). Given $R, Q>0$, then

$$
\begin{array}{rlrl}
\forall t \quad \mathbb{E}\left[P_{t}\right] & \leq B_{P_{0}} & & \lambda \in(0,1], \quad \forall P_{0} \geq 0 \\
\lim _{t \rightarrow \infty} \mathbb{E}\left[P_{t}\right]=+\infty & & \lambda=0 \text { and some } P_{0} \geq 0
\end{array}
$$

where $B_{P_{0}}$ depends on the initial condition $P_{0} \geq 0$. The inequality indicates that the matrix difference is positive semidefinite.

Theorem 3.6 (Monotonicity). Assume $m=n=1$; i.e. $x_{t}, s_{t}, y_{t}, y_{t}^{s} \in \mathbb{R}$. Given $\lambda_{1}, \lambda_{2} \in \mathbb{R}$ with $0<\lambda_{1} \leq \lambda_{2}$ then

$$
\mathbb{E}_{\lambda_{1}}\left[P_{t}\right] \leq \mathbb{E}_{\lambda_{2}}\left[P_{t}\right]
$$

Theorem 3.7 (Optimal Estimator). Assume $m=n=1$; i.e. $x_{t}, s_{t}, y_{t}, y_{t}^{s} \in \mathbb{R}$. Define

$$
\begin{array}{ll}
P_{t \mid t}^{x}:=E\left(x_{t}-\hat{x}_{t \mid t}\right)\left(x_{t}-\hat{x}_{t \mid t}\right)^{\prime} & P_{t \mid t}^{x, s}:=E\left(x_{t}-\hat{x}_{t \mid t}\right)\left(s_{t}-\hat{s}_{t \mid t}\right)^{\prime} \\
P_{t \mid t}^{s}:=E\left(s_{t}-\hat{s}_{t \mid t}\right)\left(s_{t}-\hat{s}_{t \mid t}\right)^{\prime} & P_{t \mid t}:=\left(\begin{array}{cc}
P_{t \mid t}^{x} & P_{t \mid t}^{x, s} \\
P_{t \mid t}^{x, s} & P_{t \mid t}^{s}
\end{array}\right)
\end{array}
$$




$$
\begin{gathered}
M_{t+1 \mid t}^{0}:=\left(\begin{array}{cc}
\left.C P_{t+1 \mid t}^{x} C+R\right)^{-1} & 0 \\
0 & 0
\end{array}\right) \\
M_{t+1 \mid t}^{1}:=\left(\begin{array}{cc}
C P_{t+1 \mid t}^{x} C+R & C P_{t+1 \mid t}^{x, s} \\
C P_{t+1 \mid t}^{x, s} & P_{t+1 \mid t}^{s}
\end{array}\right) \\
\tilde{A}=\left(\begin{array}{ll}
A & 0 \\
A & I
\end{array}\right) \quad \tilde{C}=\left(\begin{array}{ll}
C & 0 \\
0 & I
\end{array}\right) \quad \tilde{Q}:=Q\left(\begin{array}{ll}
1 & 1 \\
1 & 1
\end{array}\right)
\end{gathered}
$$

Then the optimal estimator $\hat{X}_{t+1 \mid t+1}$, which minimizes the mean-squared estimation error and is the MLE, and the expected error covariance $P_{t+1 \mid t+1}$ are determined by the following Kalman filter equations:

$$
\begin{aligned}
\hat{X}_{t+1 \mid t} & =\tilde{A} \hat{X}_{t \mid t} \\
P_{t+1 \mid t} & =\tilde{A} P_{t \mid t} \tilde{A}^{\prime}+\tilde{Q} \\
K_{t+1} & :=P_{t+1 \mid t} \tilde{C}^{\prime}\left[\left(1-\gamma_{t+1}\right) M_{t+1 \mid t}^{0}+\gamma_{t+1} M_{t+1 \mid t}^{1}\right] \\
\hat{X}_{t+1 \mid t+1} & =\hat{X}_{t+1 \mid t}+K_{t+1}\left(Y_{t+1}-\tilde{C} \hat{X}_{t+1 \mid t}\right) \\
P_{t+1 \mid t+1} & =\left(I-K_{t+1} \tilde{C}\right) P_{t+1 \mid t}
\end{aligned}
$$

These are the standard Kalman filter equations with missing observations. However, this theorem indicates that we have agreement with the expected equations, even though $P_{t+1 \mid t+1}$ and $\hat{X}_{t+1 \mid t+1}$ are both random variables as functions of $\gamma_{t+1}$.

\subsection{System Identification \& Inference}

Theorems 3.5, 3.6, 3.7 assume the system has been fully identified. The question remains, how does error in the system identification process affect inference. The following theorem shows that the convergence properties of the system identification algorithm imply convergence in state estimation. We begin with a few definitions - incorporating the system identification estimators (Section 3.1.2) into the variables used in state estimation (Theorem 3.7).

3.3.1 Definitions. Let $\hat{A}, \hat{C}, \hat{R}, \hat{Q}$ be as in section 3.1.2. For brevity, we group the system parameters and their estimators into the vectors

$$
\Theta:=(A, C, R, Q) \quad \hat{\Theta}:=(\hat{A}, \hat{C}, \hat{R}, \hat{Q})
$$

We note that $\hat{\Theta}$ is a function of $k: \hat{\Theta}=\hat{\Theta}(k)$, since $\hat{A}, \hat{C}, \hat{R}, \hat{Q}$ are functions of $k$; but for notational convenience we simply write $\hat{\Theta}$. Then, we modify Eqs. (19), (20), (21) by substituting in the estimators $\hat{\Theta}$ as follows:

$$
\begin{gathered}
M_{t+1 \mid t}^{0}(\hat{\Theta}):=\left(\begin{array}{cc}
\left(\hat{C} P_{t+1 \mid t}^{x} \hat{C}+\hat{R}\right)^{-1} & 0 \\
0 & 0
\end{array}\right) \\
M_{t+1 \mid t}^{1}(\hat{\Theta}):=\left(\begin{array}{cc}
\hat{C} P_{t+1 \mid t}^{x} \hat{C}+\hat{R} & \hat{C} P_{t+1 \mid t}^{x, s} \\
\hat{C} P_{t+1 \mid t}^{x, s} & P_{t+1 \mid t}^{s}
\end{array}\right) \\
\tilde{A}(\hat{\Theta}):=\left(\begin{array}{ll}
\hat{A} & 0 \\
\hat{A} & I
\end{array}\right) \quad \tilde{C}(\hat{\Theta}):=\left(\begin{array}{ll}
\hat{C} & 0 \\
0 & I
\end{array}\right) \quad \tilde{Q}(\hat{\Theta}):=\hat{Q}\left(\begin{array}{ll}
1 & 1 \\
1 & 1
\end{array}\right)
\end{gathered}
$$


Next, we modify the state and covariance update Eqs. (22)-(26) to be functions of the parameter estimators $\hat{\Theta}$. We use the notation: $Z(\hat{\Theta}):=Z^{\hat{\Theta}}$ for any function $Z(\hat{\Theta})$.

$$
\begin{aligned}
\hat{X}_{t+1 \mid t}(\hat{\Theta}) & :=\tilde{A}^{\hat{\Theta}} \hat{X}_{t \mid t}^{\hat{\Theta}} \\
P_{t+1 \mid t}(\hat{\Theta}) & :=\tilde{A}^{\hat{\Theta}} P_{t \mid t}^{\hat{\Theta}}\left(\tilde{A}^{\hat{\Theta}}\right)^{\prime}+\tilde{Q}^{\hat{\Theta}} \\
K_{t+1}(\hat{\Theta}) & :=P_{t+1 \mid t}^{\hat{\Theta}}\left(\tilde{C}^{\hat{\Theta}}\right)^{\prime}\left[\left(1-\gamma_{t+1}\right) M_{t+1 \mid t}^{0, \hat{\Theta}}+\gamma_{t+1} M_{t+1 \mid t}^{1, \hat{\Theta}}\right] \\
\hat{X}_{t+1 \mid t+1}(\hat{\Theta}) & =\hat{X}_{t+1 \mid t}^{\hat{\Theta}}+K_{t+1}^{\hat{\Theta}}\left(Y_{t+1}-\tilde{C}^{\hat{\Theta}} \hat{X}_{t+1 \mid t}^{\hat{\Theta}}\right) \\
P_{t+1 \mid t+1}(\hat{\Theta}) & :=\left(I-K_{t+1}^{\hat{\Theta}} \tilde{C}^{\hat{\Theta}}\right) P_{t+1 \mid t}^{\hat{\Theta}}
\end{aligned}
$$

Clearly Eqs. (22)-(26) and (30)-(34) agree when $\hat{\Theta} \equiv \Theta$. Thus, we have defined an end-to-end estimation algorithm: (i) first estimate the system parameters $\hat{\Theta}$ from observations and then (ii) use the estimates $\hat{\Theta}$ and the observations to estimate the latent state and covariance. The next Theorem shows that this end-to-end estimation process converges to the optimal state and covariance estimators.

Theorem 3.8 (End-to-End Convergence). Assume $A \neq 0, C \neq 0, R>0, Q>0$. Let the spectral radius of $A, \rho(A)$ be strictly less than 1 . Given $m=n=1$; i.e. $x_{t}, s_{t}, y_{t}, y_{t}^{s} \in \mathbb{R}$. Recall that $\hat{A}, \hat{C}, \hat{Q}, \hat{R}$ are all functions of $k$, where $k$ is the number of observations of aggregate state $s_{t}$. Then as $k \rightarrow \infty$, with probability one

$$
\begin{array}{rlrl}
\hat{X}_{t+1 \mid t}(\hat{\Theta}) & \rightarrow \hat{X}_{t+1 \mid t}(\Theta) & P_{t+1 \mid t}(\hat{\Theta}) & \rightarrow P_{t+1 \mid t}(\Theta) \\
K_{t+1}(\hat{\Theta}) & \rightarrow K_{t+1}(\Theta) & \hat{X}_{t+1 \mid t+1}(\hat{\Theta}) & \rightarrow \hat{X}_{t+1 \mid t+1}(\Theta) \\
P_{t+1 \mid t+1}(\hat{\Theta}) & \rightarrow P_{t+1 \mid t+1}(\Theta) &
\end{array}
$$

where the convergence is with respect to the Frobenius norm. That is, the modified Kalman filter equations (Eqs. 30-34), which are functions of the system parameter estimators and the $\left\{\gamma_{t}\right\}$, converge to the optimal state and covariance estimates (Eqs 22-26) as the number of observations $k$ of aggregate state tends to infinity. Consequently, in the limit, running our system identification procedure followed by inference yields optimal state and covariance estimation.

\section{EXPERIMENTS}

Our framework provides a method for combining alt data with occasional ground truth. The analysis suggests that receiving ground truth with any positive frequency means we can track company financials at higher frequency than published. In this section, we empirically evaluate our framework on several datasets.

First, we study the performance of the system identification method using data synthetically generated in accordance with the linear model considered. While our theoretical results guarantee consistent estimation (and unbiased for some parameters), we find that even with very few observations, our estimators have low estimation error. Next, we verify our framework using real data to estimate quarterly revenue at 34 publicly-traded companies over 306 quarters (918 months). We use an alternative dataset of real credit card transactions that provides unknown fractions of aggregate revenue per company, on a typically weekly or bi-weekly basis. We then compare our method's performance with the industry-standard Wall Street consensus benchmark. We find that our method significantly outperforms the Wall Street consensus estimates in predicting quarterly numbers.

Second, we utilize the synthetic data again to confirm the theoretical result that the estimation error in our framework decreases monotonically with more frequent access to the ground-truth; 

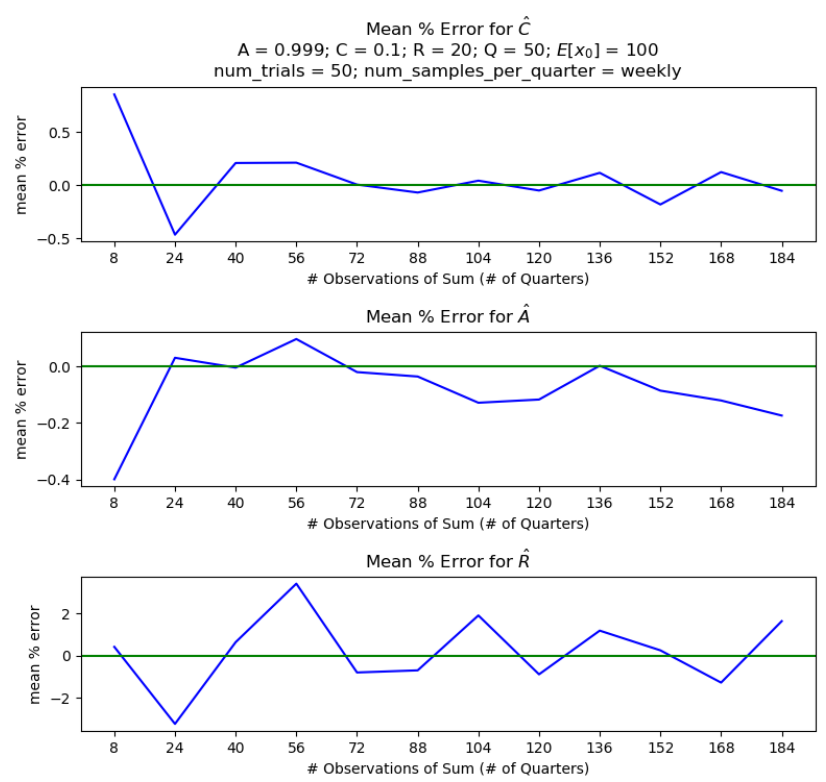

Mean \% Error for $\hat{Q}$

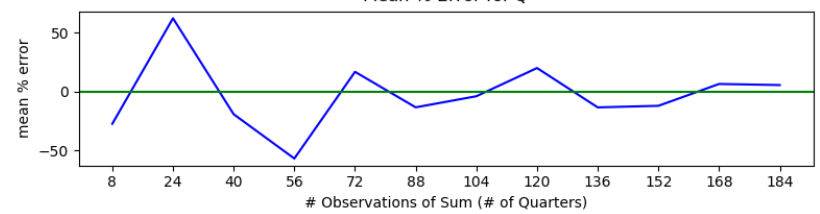

Fig. 5. Estimating $C, A, R, Q$ as a function of data set size. For a given number of quarterly observations (horizontal axis) we run 50 simulations and average the results to compute the mean percent error (blue line). The green horizontal line indicates $0 \%$ error. Since $\hat{C}$ and $\hat{R}$ are both unbiased, we see low-average-percenterror, even for small samples sizes. The estimates $\hat{A}, \hat{Q}$ quickly look unbiased as well - once the estimators $\hat{C}, \hat{R}$ have converged (see Figure 6).

and the error in the ground-truth estimation remains bounded whether the system dynamics are stable or not. We test our tracking algorithm on General Motors (GM) monthly sales data (obtained from [22]) combined with simulated alternative data to show that the higher reporting frequency leads to better estimation of the company's earnings, thus contradicting their claim in support of their decision to change from monthly to quarterly reporting in April 2018.

\subsection{System Identification with Synthetic Data}

Data: We utilize system model (1)-(2) to generate data with $n=m=1$. We utilize parameters $A=0.999, C=0.1, R=20, Q=50$ and $x_{0} \sim N(100,10)$. The choice of $A=0.999$ is done to represent the fact that usually financial systems are at the boundary of stability - they do not expand drastically, nor continually shrink. Other parameter choices are somewhat arbitrary. To mimic alt data sparsity, we generate weekly alternative data $y_{t}$, and quarterly aggregate observations of $s_{t}$. That is, for every 13 observations of $y_{t}$ we receive a single observation of $s_{t}$. For the estimators $\hat{A}$ and $\hat{Q}$, we use as input the estimated $\hat{C}, \hat{R}$. To closely mimic the modeling task with real alt data (see Section 4.2), we generate each quarter as a separate realization of a LDS with the same underlying 


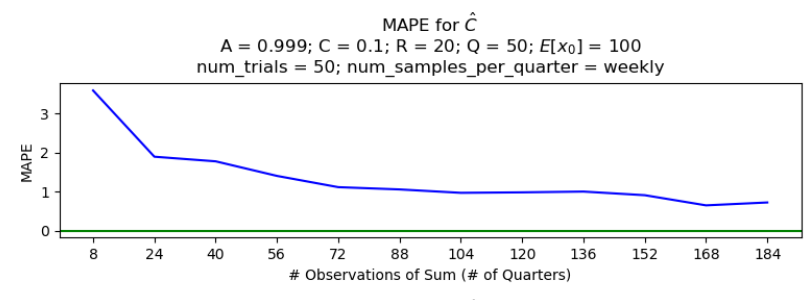

MAPE for $\hat{A}$

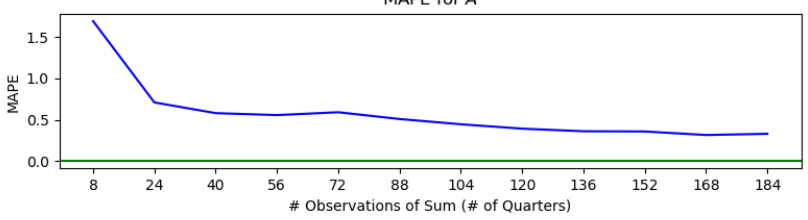

MAPE for $\hat{R}$

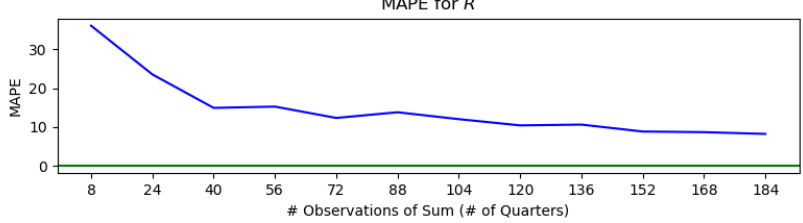

MAPE for $\hat{Q}$

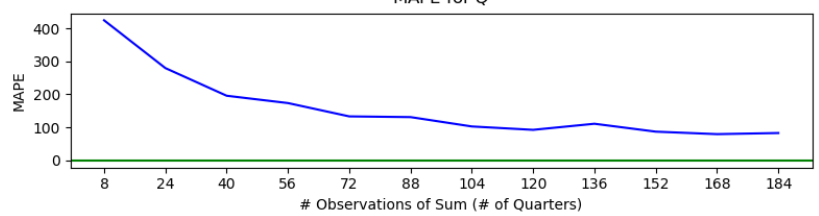

Fig. 6. MAPE for estimators $\hat{C}, \hat{A}, \hat{R}, \hat{Q}$ as a function of data set size. For a given number of quarterly observations (horizontal axis) we run 50 simulations and average the results to compute the mean-absolute percent error (blue line). The green horizontal line indicates $0 \%$ error. The mean-absolute percent error decaying empirically confirms that all the estimators are consistent.

time series. That is, 8 quarters of data would indicate that we generate 8 separate quarters of data, as opposed to a single time series running for 8 quarters.

Task: Our goal here is to estimate the underlying system parameters $A, C, Q, R$ from just the observations $y_{t}$ and the occasional, quarterly observations of $s_{t}$.

Findings: Figures 5 and 6 plot the average percent error and mean-absolute percent error (MAPE) as a function of dataset size. We find that the MAPE decays with increasing data - as would be expected from the consistency properties. And the average percent error hovers around zero, as would be expected from the unbiased properties of $\hat{C}, \hat{R}$. Our estimators for $C, R, A$ converge more rapidly than for $Q$ - which is to be expected, since $\hat{Q}$ uses the other estimators as input. Nevertheless, even for small sample sizes, we see low estimation error.

Implications: From just the observations and ocassional, infrequent summation constraint, we are able to estimate all system parameters with low error. As expected, parameter estimates look unbiased and converge as the data set size increases. And even though we use the estimated $\hat{C}, \hat{R}$ as input for $\hat{A}$ and $\hat{Q}$, a large sample size is not required for low-error estimation. 


\subsection{Forecasting with Credit Card Transactions}

4.2.1 Data. Here we use three types of data. First, we use alternative data (the $\left\{y_{t}\right\}$ ) obtained from a hedge fund. The alt data consists of aggregates of consumer credit card transactions for 34 publicly-traded retailers. ${ }^{1}$ For each company, we are provided with credit card aggregates (in dollars) at typically weekly or biweekly frequency, but no less than monthly frequency. The credit card data is a noisy estimate of company sales, as it captures only an unknown fraction of credit card spending, and it captures no cash transactions. The 34 companies are consumer-facing and have minimal business-to-business sales. For each company, we have 7-10 quarters of data in the period from fourth-quarter 2015 to first-quarter 2019. Across all companies, we have an aggregate of 306 quarters of data - with an average and median of 9 quarters of data per company.

Second, we use the actual, quarterly year-over-year (YoY) percent change in sales, obtained from a Bloomberg terminal. These are the target values we wish to predict. The YoY sales are in units of percent-change, relative to the corresponding quarter in the previous year. Since the aggregates for our model (the $s_{t}$ ) are in units of dollars, we also make use of the corresponding total quarterly sales in dollars. We convert from units of dollars $\left(s_{t}\right)$ to YoY percent change when comparing our model predictions to the benchmark - discussed next.

Finally, we make use of a third dataset: a benchmark for comparison. We use financial analyst consensus numbers, obtained from a Bloomberg Terminal for each company and quarter. The financial consensus numbers are an industry-standard, Wall St. forecasting benchmark for YoY change in quarterly sales. Analysts may, of course, utilize machine learning models in their forecasts along with expert input.

4.2.2 Task. The goal is to accurately forecast YoY quarterly sales using credit card transactions as input. The point-in-time of prediction is after the fiscal quarter ends, but before the quarterly number is publicly announced. For public companies, there is typically a multi-week gap between the end of a quarter, and the public release of that quarter's aggregate information. Thus, to forecast the quarterly report, we just need to run our tracking algorithm from the start to the end of the quarter. The point-in-time of prediction ensures that we never have access to ground truth. We then compare our estimate to the YoY Wall St. consensus number.

For each company, we use leave-one-out cross-validation on all available company data. Thus, for each of the 34 companies (with 7-10 quarters of data each), we generate 7-10 train/test splits of sizes 6-9 training quarters and 1 test quarter. The training set is used to compute per-company LDS parameters. Then, using the learned parameters, we run inference on the test sets and forecast the quarterly sales numbers. The modeling implication is that each quarter is a separate realization of a per-company LDS with the same underlying system parameters, as opposed to a single, longer time series.

4.2.3 Findings. Table 3 tabulates the results for the 306 quarters across 34 companies. In a head-to-head comparison, ours beats the benchmark in $57.2 \%$ of quarters. To determine if this is statistically significant, we consider the following hypothesis test. Let $\psi$ be the probability that our method outperforms the benchmark on a given quarter (assuming independence across quarters and companies). The null hypothesis is $\psi \leq 0.5$. The alternative hypothesis is $\psi>0.5$. We will reject the null hypothesis if we observe a $p$-value of less than $5 \cdot 10^{-2}$. For the given sample of 306 quarters, with 175 LDS wins, we obtain a $p$-value of $\sum_{i=175}^{306}\left(\begin{array}{c}306 \\ i\end{array}\right)\left(\frac{1}{2}\right)^{306}=6.9 \cdot 10^{-3}$. Thus we reject the null hypothesis with overwhelming statistical confidence.

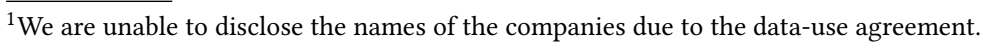




$\begin{array}{ccc}\text { Metric } & \text { LDS } & \text { Benchmark } \\ \text { RMSE } & 2.7 & 3.2 \\ \text { Median Abs Error } & 1.2 & 1.3 \\ \text { Wins (Total Quarters) } & 175 & 131 \\ \text { Win Percent } & 57.2 \% & 42.8 \%\end{array}$

Table 3. LDS versus Wall Street consensus benchmark. We learn a separate LDS model for each train/test split of each company's data. We use leave-one-out cross-validation and report the resulting test set performance. Test performance is aggregated across all 34 companies and 306 quarters. By "win" we mean a test quarter for which the LDS outperforms the benchmark. The in-general, low-percent error of the benchmark indicates the difficulty of the forecasting task. The LDS win percentage is statistically significant (see section 4.2.3).

Table 4 shows a representative sample of forecasting error for a particular company over 9 quarters. For this subset, none of the actual YoY values are zero, so we are able to compute the mean absolute percent error: $9.1 \%$ for LDS and $9.9 \%$ for the Benchmark.

4.2.4 Significance. Perhaps the most significant statistic is the win-rate of 57.2\%. [23] provides an analysis of profits at a major trading company, with a win rate of $51 \%$ - which that company successfully monetizes by trading frequently. Laughlin [23] notes that there are three types of outcomes for a trading strategy: profitable, unprofitable, and scratch (net zero profitability). Laughlin derives the probability of a profitable week given a $51 \%$ win rate, with the rest half scratch and half losses - showing that, trivially, any slight advantage becomes significant as the trading frequency increases. In the first quarter of 2015 , the $51 \%$ win rate translated into $\$ 213$ million in net trading income at this particular trading company [10]. Converting our forecasts into a trading strategy is beyond the scope of this paper, but we note that much of finance is focused on finding any forecasting edge.

4.2.5 Discussion. In a sense, it is quite surprising that our estimator works this well: each model is given at most 9 ground-truth data points to learn the system parameters, and it uses only one alternative data source of aggregated noisy credit card transactions. On the other hand, the Wall Street consensus is built on much longer history, and utilizes significant human analyst input as well as various other data sources.

In summary, our framework shows that one can extract value from high-frequency, noisy alternative data through a principled, systematic framework for tracking low frequency ground truth like quarterly revenues.

\subsection{Tracking with Synthetic Data}

Data: Next, we analyze the results of an unstable LDS. We use $A=1$ and generate synthetic ground truth and synthetic alt data - as per Eqs. (1) and (2). We the following parameters - all chosen arbitrarily: $C=2, Q=10, R=500, x_{0} \sim N(7, .1)$.

Task: Our goal here is twofold: to test the Phase Transition Theorem and the Monotonicity Theorem. First, we test that even if the alt data is undetectable and unstable, the expected error will still converge as long as $\lambda>0$. Second, we want to confirm our intuition that RMSE decreases as $\lambda$ increases.

Findings: Figure 7, top plot shows that if $\lambda=0$ the estimation error does indeed diverge, but if $\lambda$ is even slightly positive, the estimation error remains bounded. This is what we expect from the Phase Transition Theorem. The bottom plot shows that as $\lambda$ increases, the RMSE decreases - which 


$\begin{array}{ccc}\text { Quarter } & \text { LDS Abs Error(\%) } & \text { Benchmark Abs Error(\%) } \\ 1 & 22.4 \% & 16.2 \% \\ 2 & 9.1 \% & 1.5 \% \\ 3 & 4.4 \% & 5.8 \% \\ 4 & 5.6 \% & 6.1 \% \\ 5 & 18.4 \% & 11.8 \% \\ 6 & 3.0 \% & 18.2 \% \\ 7 & 4.6 \% & 2.2 \% \\ 8 & 8.3 \% & 16.7 \% \\ 9 & 5.9 \% & 10.2 \%\end{array}$

Table 4. Sample results for one company over 9 quarters. In this instance, the LDS model outperforms the benchmark in 5/9 quarters. We highlight in green (and bold) the quarters for which the LDS model outperforms the benchmark. Overall, the mean-absolute percent error for LDS here is $9.1 \%$ versus $9.9 \%$ for the benchmark.
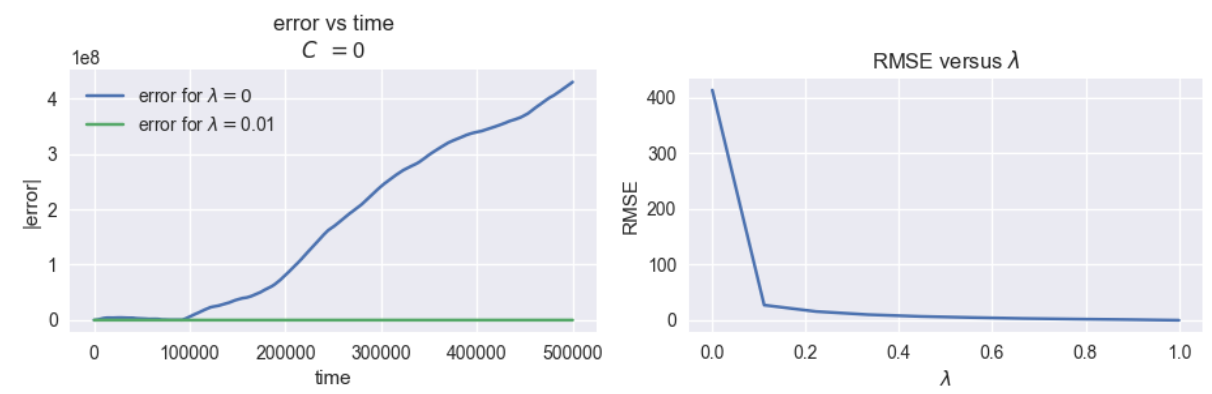

Fig. 7. The top plot shows the error for an unobserable alt data system for two values of $\lambda$. As long as $\lambda>0$, the system is trackable. But if $\lambda=0$ the error diverges. The bottom plot shows the decay in RMSE as $\lambda$ increases. For the bottom plot, we take the average RMSE over 30 simulations for each particular value of $\lambda$.

is what we expect as a consequence of the Monotonicity Theorem. Each point in the bottom plot is generated as the average over 30 simulations for each particular value of $\lambda$.

Implications: Our results empirically confirm the two main inference theorems of the paper. Furthermore, the top plot in Figure 7 suggests that companies looking to prevent analysts from tracking certain financials should (i) never make disclosures with any frequency and (ii) try and ensure that any alt data source has undetectable characteristics.

\subsection{GM Experiments}

Data: We test our algorithm on GM monthly U.S. vehicle sales. The data, compiled by [22], provides the total number of car, crossover, and trucks sold by GM each month - from January 2010 through March 2018. Starting from the monthly sales, we generate two data sets: sparse ground truth and synthetic alt data. First, we generate a sparse version of the monthly sales data by removing every second month and then every fourth month from the data. In other words, the first half of the dataset has $\lambda=0.5$ and then for the second half we use $\lambda=0.25$. Thus, we are replacing the Bernoulli process $\left\{\gamma_{t}\right\}$ with a periodic one. And then we are decreasing the frequency by half. For the second data set, we generate synthetic alt data $\left(y_{t}\right)$ as per (1) and (2) - where we set $x_{t}$ as 

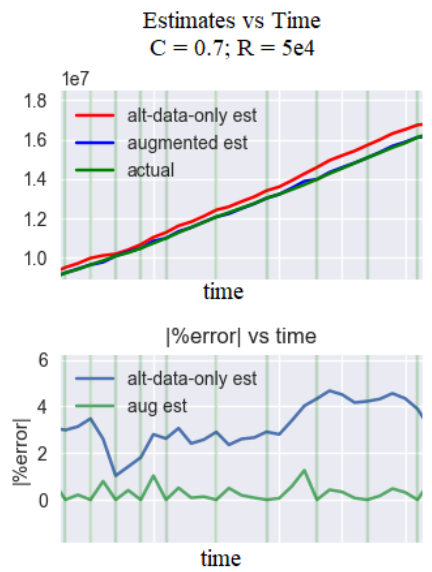

P_t (for sum) vs time

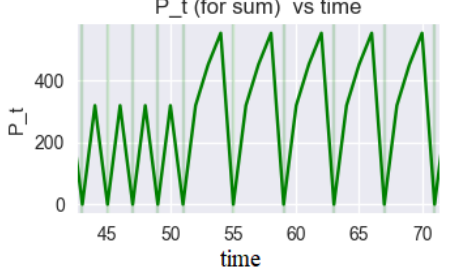

Fig. 8. Error, covariance, and estimates using our method on General Motors data combined with synthetic alt data. Our estimator takes as input (i) GM sales data every other month and then every fourth month and (ii) a simulated, monthly alt dataset. The top figure shows the error over time for our estimator (utilizing alt data and occasional aggregates) as compared with a Kalman filter on the alt data only. The middle figure shows the corresponding estimation error. The vertical green lines indicate the times when ground-truth aggregate information is made available to our model. The bottom figure shows the associated covariance for our estimator.

GM monthly ground truth. The simulated alt data uses the following parameters: $C=0.7$ (chosen arbitrarily) and $R=5 e 4$ (quite noisy). We use $A=1$ estimated from a GM training set consisting of the first 24 months (2010-2011); where for $A$ estimation we use the least squares estimation technique for auto-regressive processes described in Hamilton [14] (Chapter 4).

Task: Our goal here is twofold. First, we test GM's assertion [29] that a lower reporting frequency makes it easier to track monthly sales. And second, we validate that our framework for incorporating periodic ground truth outperforms a Kalman filter using only the alt data source.

Findings: Our experiments here disprove the hypothesis asserted by GM in [29], that a lower reporting frequency makes it easier to track sales. Instead, Figure 8 shows that a higher $\lambda$ corresponds to lower estimation error, as we would intuitively expect. In Figure 8 we also see that the error is periodic - with the minima corresponding to the times that ground-truth is made available. This makes intuitive sense - and highlights that the covariance is a function of when we observe $s_{t}$ and not the realized values. The middle plot of Figure 8 shows that our estimator tracks ground truth much more closely than if we ran a standard Kalman filter over just the alt data.

Implications: Our findings suggest to policy makers that the frequency of required reporting affects company transparency in a quantifiable way. Furthermore, we validate that our technique can usefully track company financials at higher frequency than published - by making use of an alt data source.

\section{CONCLUSION}

In this paper, we considered the problem of forecasting fine-grained company financials using alternative data. We utilized the classical framework of linear systems to address this question. We solved the two key problems: (i) system identification or model parameter learning and (ii) optimal inference of state using observed data. Specifically, our system identification algorithm 
provides simple, consistent estimators for all model parameters; and unbiased estimation for some of the parameters. Our inference algorithm is a natural extension of the classical Kalman filtering (or Belief Propagation) algorithm. Using synthetic data, we found that our asymptotic analytic results are quite sharp. Using real-data, we showed that credit card transaction data for 34 public companies can be utilized in estimating quarterly revenue more accurately than compared to the Wall Street consensus estimates obtained from Bloomberg. Using our inference algorithm, we found GM to have made a contradictory claim; we concluded that a decrease in reporting frequency should not have a positive affect on trackability [29].

Our forecasting and tracking framework is not limited to transactions data. For example, suppose we want to forecast a car factory's output. If recently-manufactured cars are stored outside, then through weekly (say) satellite images [53] we might observe an unknown fraction of the factory's output. Then, if historical disclosures of output are available (e.g. from quarterly reports), our data looks like a frequent proxy signal for intermittent, aggregate ground truth. If we approximate factory production and satellite observations of it as noisy, linear processes, then our methods apply, and we can make full use of our foresting and tracking framework. Of course, experimental analysis would be needed to verify the utility of the linear approximation.

\section{ACKNOWLEDGMENTS}

This work was supported in parts by NSF projects CMMI-1462158, CMMI-1634259, CNS-1523546, TRIPODS Phase 1; a joint project with KAIST (South Korea); and a project funded by KACST.

\section{REFERENCES}

[1] Eagle Alpha. 2018. Eagle Alpha Alternative Data Use Cases. https://eaglealpha.com/eagle-alphas-alternative-data-usecases. Accessed: 2018-05-10.

[2] AlternativeData.org. 2018. Alternative Data by the Numbers. https://alternativedata.org/resources/alternative-data-bythe-numbers. Accessed: 2018-05-17.

[3] AlternativeData.org. 2018. Alternative Data Database. https://alternativedata.org/data-providers/. Accessed: 2018-1110.

[4] Harry Asada. 2018. MIT 2.160. Lecture Notes.

[5] Dimitri P Bertsekas. 1995. Dynamic programming and optimal control. Vol. 1. Athena scientific, Belmont, MA.

[6] Johan Bollen, Huina Mao, and Xiaojun Zeng. 2011. Twitter mood predicts the stock market. fournal of Computational Science 2, 1 (2011), 1 - 8 .

[7] Zhengping Che, Sanjay Purushotham, Kyunghyun Cho, David Sontag, and Yan Liu. 2018. Recurrent Neural Networks for Multivariate Time Series with Missing Values. Nature, Scientific Reports 8, 6085 (April 2018).

[8] Ryan Dezember. 2018. Your Smartphone’s Location Data Is Worth Big Money to Wall Street. https://www.wsj.com/ articles/your-smartphones-location-data-is-worth-big-money-to-wall-street-1541131260. Accessed: 2018-11-04.

[9] Amir Efrati. 2018. U.S. Slowdown at Uber and Lyft. https://www.theinformation.com/articles/ u-s-slowdown-at-uber-and-lyft. Accessed: 2018-10-25.

[10] Virtu Financial. 2015. Virtu Financial Announces First Quarter 2015 Results. http://ir.virtu.com/financials-and-filings/ quarterly-results/default.aspx Accessed: 2019-07-10.

[11] Graham C. Goodwin and Kwai Sang Sin. 1984. Adaptive Filtering Prediction and Control. Dover Publications, Mineola, NY.

[12] N. J. Gordon, D. J. Salmond, and A. F. M. Smith. 1993. Novel approach to nonlinear/non-Gaussian Bayesian state estimation. IEE Proceedings F - Radar and Signal Processing 140, 2 (April 1993), 107-113.

[13] Nachi Gupta. 2006. Kalman Filtering in the Presence of State Space Equality Constraints. In 2007 Chinese Control Conference. IEEE, 107-113.

[14] James Douglas Hamilton. 1994. Time series analysis. Princeton Univ. Press, Princeton, NJ.

[15] P. D. Hanlon and P. S. Maybeck. 2000. Characterization of Kalman filter residuals in the presence of mismodeling. IEEE Trans. Aerospace Electron. Systems 36, 1 (Jan 2000), 114-131.

[16] Moritz Hardt, Tengyu Ma, and Benjamin Recht. 2016. Gradient Descent Learns Linear Dynamical Systems. arXiv:arXiv:1609.05191

[17] Elad Hazan, Holden Lee, Karan Singh, Cyril Zhang, and Yi Zhang. 2018. Spectral Filtering for General Linear Dynamical Systems. arXiv:arXiv:1802.03981 
[18] Elad Hazan, Karan Singh, and Cyril Zhang. 2017. Learning Linear Dynamical Systems via Spectral Filtering. In Advances in Neural Information Processing Systems 30. Curran Associates, Inc., 6702-6712.

[19] Nicholas Hensel. 2014. Development of a Human Body Upper Arm Dynamic Model for Compensation and Control of a Body Mounted Robot. Master's thesis. Massachusetts Institute of Technology.

[20] Bradley Hope. 2015. Provider of Personal Finance Tools Tracks Bank Cards Sells Data to Investors. https://www. wsj.com/articles/provider-of-personal-finance-tools-tracks-bank-cards-sells-data-to-investors-1438914620. Accessed: 2018-05-10.

[21] Mahdi Karami, Martha White, Dale Schuurmans, and Csaba Szepesvari. 2017. Multi-view Matrix Factorization for Linear Dynamical System Estimation. In Advances in Neural Information Processing Systems 30, I. Guyon, U. V. Luxburg, S. Bengio, H. Wallach, R. Fergus, S. Vishwanathan, and R. Garnett (Eds.). Curran Associates, Inc., 7092-7101.

[22] Knoema. 2018. U.S. Auto Sales, Monthly Update. https://knoema.com/MIAS2017/u-s-auto-sales-monthly-update. Accessed: 2018-05-07.

[23] Greg Laughlin. 2014. Insights Into High Frequency Trading from the Virtu Initial Public Offering. http://online.wsj. com/public/resources/documents/VirtuOverview.pdf. Accessed: 2019-04-30.

[24] Lennart Ljung. 1999. System Identification. Theory for the User. Second Edition. PTR Prentice Hall, Upper Saddle River, NJ.

[25] Philippe Rigollet and Jan-Christian Hutter. 2017. Lecture notes in High Dimensional Statistics. http://www-math.mit edu/ rigollet/PDFs/RigNotes17.pdf Accessed: 2018-05-07.

[26] D. Simon and Tien Li Chia. 2002. Kalman filtering with state equality constraints. IEEE Trans. Aerospace Electron. Systems 38, 1 (Jan 2002), 128-136.

[27] B. Sinopoli, L. Schenato, M. Franceschetti, K. Poolla, M. I. Jordan, and S. S. Sastry. 2004. Kalman filtering with intermittent observations. IEEE Trans. Automat. Control 49, 9 (Sept 2004), 1453-1464.

[28] E. A. Wan and R. Van Der Merwe. 2000. The unscented Kalman filter for nonlinear estimation. In Proceedings of the IEEE 2000 Adaptive Systems for Signal Processing, Communications, and Control Symposium. IEEE, 153-158.

[29] Joseph White. 2018. GM to drop monthly U.S. vehicle sale reports. https://www.reuters.com/article/ us-usa-autos-gm/gm-to-drop-monthly-u-s-vehicle-sale-reports-idUSKCN1HA0C9. Accessed: 2018-05-07.

[30] Robin Wigglesworth. 2018. Asset management's fight for alternative data analysts heats up. https://www.ft.com/ content/2f454550-02c8-11e8-9650-9c0ad2d7c5b5. Accessed: 2018-05-07.

[31] Robin Wigglesworth. 2018. Asset managers double spending on new data in hunt for edge. https://www.ft.com/ content/3c321c14-52d4-11e8-b24e-cad6aa67e23e. Accessed: 2018-05-10.

\section{A APPENDIX}

Here we provide the proofs of our results in system identification and inference. We start in Section A.1 by proving the unbiased, consistency, and finite sample properties for system identification. Then in Section A.2 for inference, we prove the Phase Transition Theorem in the vector case. We provide a short proof of the Monotonicity Theorem, along with any needed lemmas. Finally, we prove the End-To-End Convergence Theorem in Appendix A.3.

\section{A.1 System Identification Proofs}

We now derive estimators for all system parameters: $A, C, Q, R$.

A.1.1 Proof of Theorem 3.1. We convert the problem of estimating $C$ into a multidimensional linear regression problem, i.e. $C$ is the unknown model parameter that we wish to estimate based on the observations of the associated linear regression problem. Subsequently, using the classical theory of linear regression, this will allow us to establish that the ordinary least squares (OLS) estimation of the corresponding regression problem will be both an unbiased and consistent estimate of $C$. Further, we will be able to provide finite-sample analysis. To that end, recall $S_{i}:=\sum_{j=t_{i-1}+1}^{t_{i}} x_{j}$. Using (3), it follows that for $1 \leq i \leq k$,

$$
C S_{i}+\sum_{j=t_{i-1}+1}^{t_{i}} v_{j}=\sum_{j=t_{i-1}+1}^{t_{i}} y_{j} .
$$


Define

$$
\tilde{V}_{i}:=\frac{1}{\sqrt{t_{i}-t_{i-1}}} \sum_{j=t_{i-1}+1}^{t_{i}} v_{j}
$$

That is,

$$
\mathbb{E}\left[\tilde{V}_{i}\right]=0, \quad \mathbb{E}\left[\tilde{V}_{i} \tilde{V}_{i}^{\prime}\right]=R
$$

Using definitions from (6), (7), (36) in (35), we obtain

$$
\left[\begin{array}{c}
-\tilde{S}_{1}- \\
\vdots \\
-\tilde{S}_{k}-
\end{array}\right]\left[\begin{array}{ccc}
\mid & & \mid \\
C_{1} & \ldots & C_{m} \\
\mid & & \mid
\end{array}\right]+\left[\begin{array}{c}
-\tilde{V}_{1}- \\
\vdots \\
-\tilde{V}_{k}-
\end{array}\right]=\left[\begin{array}{c}
-\tilde{Y}_{1}- \\
\vdots \\
-\tilde{Y}_{k}-
\end{array}\right]
$$

For finite sample analysis, we require that the $\left\{v_{t}\right\}$ are sub-Gaussian, which implies that the $\tilde{V}_{i}$ are sub-Gaussian. Then Eq. (38) represents $m$ linear regressions where the goal is to learn model parameters $C_{1}, \ldots, C_{m}$ which are each $n$ dimensional. Let us consider one of these - let $\hat{C}_{1}$ be the OLS estimate of $C_{1}$. From definition (9), we have the $k \times n$ matrix

$$
\tilde{S}=\left[\begin{array}{c}
-\tilde{S}_{1}- \\
\vdots \\
-\tilde{S}_{k}-
\end{array}\right]
$$

Then, using standard results on linear regression (cf. see Theorem 2.2 and Remark 2.3 [25]), it follows that

$$
\left\|\hat{C}_{1}-C_{1}\right\|_{2}^{2} \leq c\|R\|_{\infty, \infty} \frac{n+\log (1 / \delta)}{k \lambda_{\min }\left(\frac{1}{k} \tilde{S}^{T} \tilde{S}\right)}
$$

with probability at least $1-\delta$, where $\lambda_{\min }\left(\frac{1}{k} \tilde{S}^{T} \tilde{S}\right)$ is the smallest eigenvalue of symmetric matrix $\frac{1}{k} \tilde{S}^{T} \tilde{S}$, and $c>0$ is a universal constant. The same argument holds true for $\hat{C}_{i}$ for all $i, i \leq m \leq k$. Therefore, with choice of $\delta=1 /\left(m^{2} n k^{2}\right)$ and union bound, it follows that

$$
\|\hat{C}-C\|_{F}^{2} \leq c\|R\|_{\infty, \infty} \frac{n+\log n+\log m+\log k}{k \lambda_{\min }\left(\frac{1}{k} \tilde{S}^{T} \tilde{S}\right)},
$$

with probability at least $1-1 /\left(m n k^{2}\right)$.

Now let the spectral radius of $A, \rho(A)$ be strictly less than 1 . Then, the resulting stochastic process $x_{t}$ is stationary and ergodic. Therefore, $\lambda_{\min }\left(\frac{1}{k} \tilde{S}^{T} \tilde{S}\right) \rightarrow \lambda_{\min }(M)$ with probability 1 as $k \rightarrow \infty$, where $M=\mathbb{E}\left[x x^{\prime}\right]$ where $x$ is distributed per stationary distribution of the stochastic process $x_{t}, t \geq 1$. And $\lambda_{\min }(M)>0$. Therefore, from (41) it follows that $\hat{C} \rightarrow C$ with probability 1 as $k \rightarrow \infty$.

Assumption 8.3 of [14] is satisfied under the setup of our interest. Therefore, it follows that the estimator $\hat{C}$ is unbiased. That is, $\mathbb{E}[\hat{C}]=C$. This completes the proof of Theorem 3.1.

A.1.2 Proof of Theorem 3.2. Theorem 3.1 establishes that $\hat{C} \rightarrow C$ almost surely. The matrix $R$ is noise covariance matrix associated with the (multiple) linear regression setup. Further, the noise terms are i.i.d. and independent of all other variables. Therefore, by classical theory of linear regression it follows that since $\hat{C}$ is the solution of the associated ordinary least squares (with $k \geq \max (m, n)$ ), we have that $\hat{R}$ defined by (12) is an unbiased estimator of $R$ as per Chapter 8 [14]. To establish $\hat{R} \rightarrow R$ almost surely, we note further that the noise random variable has bounded fourth moment (actually all moments due to Gaussian distribution). Then again by [14](Chapter 8), $\hat{R} \rightarrow R$ almost surely. 
A.1.3 Proof of Theorem 3.3. The proof follows arguments similar to those in the proof of Theorem 3.1. However it is somewhat more involved. To that end, we start by considering the scenario where $\hat{C}=C$, i.e $C$ is known. Recall $S_{i}:=\sum_{j=t_{i-1}+1}^{t_{i}} x_{j}$. From (3), it follows

$$
\begin{aligned}
& A S_{1}=A \sum_{j=0}^{t_{1}} x_{j}=\sum_{j=1}^{t_{1}+1}\left(x_{j}-w_{j}\right)=S_{1}+x_{t_{1}+1}-x_{0}-\sum_{j=1}^{t_{1}+1} w_{j} \\
& \Longrightarrow(I-A) S_{1}=\sum_{j=1}^{t_{1}+1} w_{j}+x_{0}-x_{t_{1}+1}
\end{aligned}
$$

By hypothesis, $C$ has a left-inverse $C^{-1}$. Then

$$
\begin{aligned}
(I-A) S_{1}-C^{-1} y_{0}+A C^{-1} y_{t_{1}} & =\sum_{j=1}^{t_{1}+1} w_{j}-C^{-1} v_{0}+A C^{-1} v_{t_{1}}-w_{t_{1}+1} \\
& =\sum_{j=1}^{t_{1}} w_{j}+A C^{-1} v_{t_{1}}-C^{-1} v_{0}
\end{aligned}
$$

Normalizing:

$$
\frac{1}{\sqrt{t_{1}}}(I-A) S_{1}+A \frac{C^{-1}}{\sqrt{t_{1}}} y_{t_{1}}+\tilde{W}_{1}=\frac{1}{\sqrt{t_{1}}} C^{-1} y_{0}
$$

where

$$
\tilde{W}_{i}:=\frac{-\sum_{j=t_{i-1}+2}^{t_{i}} w_{j}}{\sqrt{t_{i}-t_{i-1}-1}}+\frac{\left(C^{-1} v_{t_{i-1}+1}-A C^{-1} v_{t_{i}}\right)}{\sqrt{t_{i}-t_{i-1}-1}}
$$

Thus,

$$
\begin{aligned}
\mathbb{E}\left[\tilde{W}_{i}\right] & =0 \\
\text { and } \quad \mathbb{E}\left[\tilde{W}_{i} \tilde{W}_{i}^{\prime}\right] & =Q+\frac{C^{-1} R\left(C^{-1}\right)^{\prime}+A C^{-1} R\left(A C^{-1}\right)^{\prime}}{t_{i}-t_{i-1}-1}
\end{aligned}
$$

For each $i$, Eq. (42) becomes

$$
A \frac{\left(C^{-1} y_{t_{i}}-S_{i}\right)}{\sqrt{t_{i}-t_{i-1}-1}}+\tilde{W}_{i}=\frac{C^{-1} y_{t_{i-1}+1}-S_{i}}{\sqrt{t_{i}-t_{i-1}-1}}
$$

By hypothesis, we observe $S_{i}$. So if $C$ is known, then the right-hand side of Eq. (45) can be computed from the observations. And the left-hand side of Eq. (45) is a linear function of $A$ with additive, mean-zero noise $\tilde{W}_{i}$. Furthermore, since the time between observations of $s_{t}$ is constant, the noise $\tilde{W}_{i}$ is homoskedastic. Thus, we can solve for $A$ with multiple, multivariate regression. In detail, let $A_{j}$ denote the $j$-th row of $A$. Then substituting (8), equation (45) becomes

$$
\left[\begin{array}{c}
-D_{1}- \\
\vdots \\
-D_{k}-
\end{array}\right]\left[\begin{array}{ccc}
\mid & & \mid \\
A_{1} & \ldots & A_{n} \\
\mid & & \mid
\end{array}\right]+\left[\begin{array}{c}
-\tilde{W}_{1}- \\
\vdots \\
-\tilde{W}_{k}-
\end{array}\right]=\left[\begin{array}{c}
-G_{1}- \\
\vdots \\
-G_{k}-
\end{array}\right]
$$

Eq. (46) is a linear matrix equation with mean-zero, independent, homoscedastic noise. Therefore, using arguments similar to those utilized in the proof of Theorem 3.1, it follows that

$$
\|\hat{A}-A\|_{F}^{2} \leq \gamma \frac{n+\log n+\log m+\log k}{k \lambda_{\min }\left(\frac{1}{k} \tilde{D}^{T} \tilde{D}\right)},
$$


with probability at least $1-1 /\left(m n k^{2}\right)$. Further, with the spectral radius of $\rho(A)$ strictly less than 1 , we have that the stochastic processes $x_{t}, y_{t}$ are stationary and ergodic. Therefore, it follows that $\lambda_{\text {min }}\left(\frac{1}{k} \tilde{D}^{T} \tilde{D}\right) \rightarrow \lambda_{\text {min }}(Z)$ with probability 1 as $k \rightarrow \infty$, where $Z$ is an appropriate positive definite matrix. Therefore, from (47) it follows that $\hat{A} \rightarrow A$ with probability 1 as $k \rightarrow \infty$. This almost sure convergence assumes that $\hat{C}=C$. That is, we have established that $\hat{A}(C) \rightarrow A$ as $k \rightarrow \infty$ with probability 1.

Now $\hat{A}(\hat{C}) \rightarrow \hat{A}(C)$ as $\hat{C} \rightarrow C$ since $\hat{A}$ is solution of ordinary least squares (or well-conditioned convex optimization, for $k$ large enough). By Theorem 3.1, we have established that $\hat{C} \rightarrow C$ almost surely. Therefore, we conclude that $\hat{A}(\hat{C}) \rightarrow A$ almost surely as $k \rightarrow \infty$. This completes the proof of consistency of $\hat{A}(\hat{C})$.

A.1.4 Proof of Theorem 3.4. As argued already, $\hat{C}, \hat{R}, \hat{A}$ converge almost surely to $C, R$ and $A$ respectively. Now in the proof of Theorem 3.3, we constructed a linear matrix Equation (46) where we utilized knowledge of $C$. The independent noise term in these equations correspond to $\tilde{W}_{i}$ which is zero mean and has covariance that obeys (43), (44). Now the fourth moments of $\left\{\tilde{W}_{i}\right\}$ exist since the $\left\{v_{i}\right\}$ and $\left\{w_{i}\right\}$ are all independent with finite fourth moments. Therefore, by [14](Chapter 8), it is feasible to obtain a consistent estimator of $\mathbb{E}\left[\tilde{W}_{i} \tilde{W}_{i}^{\prime}\right]$. Since we have consistent estimators for $C, R$ and $A$, we can consistently estimate $Q$. And this is precisely given by (14). This completes the proof of Theorem 3.4.

\section{A.2 Inference Proofs}

A.2.1 Proof of Theorem 3.7. The optimal estimator $\hat{X}_{t+1 \mid t}$ minimizes the mean-squared estimation error and is in fact the MLE. We start by showing that we can embed the occasional observation of $s_{t}$ as an almost-noiseless observation in the context of a linear dynamical system. We will then modify the classical Kalman filtering equations to derive the MLE. In contrast to the system identification sections, here we allow $\left\{\gamma_{t}\right\}$ to follow a Bernoulli process - as opposed to a non-random, periodic sequence (see Section 2.2). Thus, we need to modify Eq. (2). Let

$$
y_{t}^{s}=s_{t}+v_{t}^{s}
$$

We set $v_{s}^{t}$ to be a Gaussian random vector with zero mean and:

$$
\mathbb{E}\left[\left(v_{t}^{s}\right)\left(v_{t}^{s}\right)^{\prime}\right]=\left\{\begin{array}{llll}
\epsilon I & \text { if } & \gamma_{t}=1 & \text { (observed) } \\
\sigma^{2} I & \text { if } & \gamma_{t}=0 \text { (not observed) }
\end{array}\right.
$$

where $I$ is the $n \times n$ identity matrix, and $\left\{\gamma_{t}\right\}$ is the Bernoulli process with parameter $0 \leq \lambda \leq 1$. We assume $\epsilon \approx 0$, and $\sigma \gg 1$. If we take the limits $\sigma \rightarrow \infty$, and $\epsilon \rightarrow 0$, then $\gamma_{t}=1$ indicates that we observe $s_{t}$ at time $t$; and $\gamma_{t}=0$ indicates otherwise. We start by writing

$$
X_{t+1}=\tilde{A} X_{t}+W_{t} \quad Y_{t}=\tilde{C} X_{t}+V_{t}
$$

where:

$$
\begin{gathered}
\tilde{A}=\left(\begin{array}{ll}
A & 0 \\
A & I
\end{array}\right) \quad \tilde{C}=\left(\begin{array}{ll}
C & 0 \\
0 & I
\end{array}\right) \quad W_{t}=\left(\begin{array}{l}
1 \\
1
\end{array}\right) w_{t} \\
V_{t}=\left(\begin{array}{l}
v_{t} \\
v_{t}^{s}
\end{array}\right) \quad \tilde{Q}:=\mathbb{E}\left[W_{t} W_{t}^{\prime}\right]=Q\left(\begin{array}{ll}
1 & 1 \\
1 & 1
\end{array}\right) \\
\tilde{R}_{t}:=\mathbb{E}\left[V_{t} V_{t}^{\prime}\right]=\left(\begin{array}{cc}
R & 0 \\
0 & I\left[\gamma_{t} \epsilon+\left(1-\gamma_{t}\right) \sigma^{2}\right]
\end{array}\right)
\end{gathered}
$$


The $\left\{W_{t}\right\}$ and $\left\{V_{t}\right\}$ are independent Gaussian random vectors with zero mean and covariance matrices as above. Note that $\tilde{R}_{t}$ is a random variable - as a function of $\gamma_{t}$.

The system equations (Eq. 48) represent a linear dynamical system with Gaussian noise. Here, as contrasted with classical Kalman filtering, components of the observation noise may have infinite variance depending on $\gamma_{t}$. By contrast, [27] considers the case where the observation noise components are all of finite variance or all of infinite variance. It remains to compute the gain $K_{t+1}$. First:

$$
\begin{aligned}
& \tilde{C} P_{t+1 \mid t} \tilde{C}^{\prime}+\tilde{R}_{t+1}= \\
& \left(\begin{array}{cc}
C P_{t+1 \mid t}^{x} C+R & C P_{t+1 \mid t}^{x, s} \\
C P_{t+1 \mid t}^{x, s} & P_{t+1 \mid t}^{s}+I\left[\gamma_{t+1} \epsilon+\left(1-\gamma_{t+1}\right) \sigma^{2}\right]
\end{array}\right)
\end{aligned}
$$

where for estimators $\hat{X}_{t \mid t}, \hat{s}_{t \mid t}$ we recall

$$
\begin{aligned}
P_{t \mid t}^{x} & :=E\left(x_{t}-\hat{x}_{t \mid t}\right)\left(x_{t}-\hat{x}_{t \mid t}\right)^{\prime} \\
P_{t \mid t}^{s} & :=E\left(s_{t}-\hat{s}_{t \mid t}\right)\left(s_{t}-\hat{s}_{t \mid t}\right)^{\prime} \\
P_{t \mid t}^{x, s} & :=E\left(x_{t}-\hat{x}_{t \mid t}\right)\left(s_{t}-\hat{s}_{t \mid t}\right)^{\prime}
\end{aligned} \quad P_{t \mid t}:=\left(\begin{array}{cc}
P_{t \mid t}^{x} & P_{t \mid t}^{x, s} \\
P_{t \mid t}^{x, s} & P_{t \mid t}^{s}
\end{array}\right)
$$

To compute the gain $K_{t}$ we need to invert Eq. (49). There are two cases. First, if $\gamma_{t+1}=0$, then $s_{t+1}$ is not observed, and we compute the inverse of Eq. (49) by taking the limit $\sigma \rightarrow \infty$ :

$$
\begin{aligned}
M_{t+1 \mid t}^{0} & :=\lim _{\sigma \rightarrow \infty}\left[\tilde{C} P_{t+1 \mid t} \tilde{C}^{\prime}+\tilde{R}_{t+1}\right]^{-1} \\
& =\left(\begin{array}{cc}
\left(C P_{t+1 \mid t}^{x} C+R\right)^{-1} & 0 \\
0 & 0
\end{array}\right)
\end{aligned}
$$

In the second case, $\gamma_{t+1}=1$, so $s_{t+1}$ is observed, and all entries of $\tilde{R}_{t+1}$ are finite. Then:

$$
\begin{aligned}
M_{t+1 \mid t}^{1} & \left.:=\left[\tilde{C} P_{t+1 \mid t} \tilde{C}^{\prime}+\tilde{R}_{t+1}^{\prime}\right)\right]^{-1} \\
& =\left(\begin{array}{cc}
C P_{t+1 \mid t}^{x} C+R & C P_{t+1 \mid t}^{x, s} \\
C P_{t+1 \mid t}^{x, s} & P_{t+1 \mid t}^{s}
\end{array}\right)^{-1}
\end{aligned}
$$

Combining the above, we have:

$$
K_{t+1}=P_{t+1 \mid t} \tilde{C}^{\prime}\left[\left(1-\gamma_{t+1}\right) M_{t+1 \mid t}^{0}+\gamma_{t+1} M_{t+1 \mid t}^{1}\right]
$$

Note that the case $\gamma_{t}=0$ corresponds to performing an update where the $y_{t}^{s}$ components of $y_{t}$ are ignored. The result follows.

We have thus derived the desired optimal estimator by modifying the Kalman filtering equations. This algorithm corresponds to Gaussian belief propogation but where the graph structure is a function of the realized $\left\{\gamma_{t}\right\}$. Next we present a modified version of the Riccati equation which we will be needed for proving the main inference theorems of the paper.

A.2.2 Modified Riccati Equation. Here we derive a variation of the classical algebraic Riccati equation, which will be needed for proving the Phase Transition Theorem.

To start, we expand Eq. (23) with (26), and then apply (24):

$$
\begin{aligned}
P_{t+1 \mid t}= & \tilde{A}\left(1-K_{t} \tilde{C}\right) P_{t \mid t-1} \tilde{A}^{\prime}+\tilde{Q} \\
= & \tilde{A} P_{t \mid t-1} \tilde{A}^{\prime}+\tilde{Q} \\
& -\tilde{A} P_{t \mid t-1} \tilde{C}\left[\left(1-\gamma_{t}\right) M_{t \mid t-1}^{0}+\gamma_{t} M_{t \mid t-1}^{1}\right] \tilde{C}^{\prime} P_{t \mid t-1} \tilde{A}^{\prime}
\end{aligned}
$$


where $M_{t \mid t-1}^{0}$ and $M_{t \mid t-1}^{1}$ are as in Eqs. (50) and (51) respectively; and both are deterministic functions of $P_{t+1 \mid t}$. To simplify notation we define:

$$
P_{t}=P_{t \mid t-1} \quad M_{t}^{0}=M_{t \mid t-1}^{0} \quad M_{t}^{1}=M_{t \mid t-1}^{1}
$$

Then:

$$
P_{t+1}=\tilde{A} P_{t} \tilde{A}^{\prime}+\tilde{Q}-\tilde{A} P_{t} \tilde{C}\left[\left(1-\gamma_{t}\right) M_{t}^{0}+\gamma_{t} M_{t}^{1}\right] \tilde{C}^{\prime} P_{t} \tilde{A}^{\prime}
$$

If steady state exists, it will satisfy the following modified algebraic Riccati equation:

$$
g_{\lambda}(X)=\tilde{A} X \tilde{A}^{\prime}+\tilde{Q}-\tilde{A} X \tilde{C}\left[(1-\lambda) M^{0}(X)+\lambda M^{1}(X)\right] \tilde{C}^{\prime} X \tilde{A}^{\prime}
$$

where $X=\left(\begin{array}{ll}X_{1,1} & X_{1,2} \\ X_{1,2} & X_{2,2}\end{array}\right)$ is a block symmetric, PSD matrix with $X_{2,2}>0$ (since $\epsilon>0$ ) and:

$$
\begin{aligned}
& M^{0}(X)=\left(\begin{array}{cr}
\left(C X_{1,1} C+R\right)^{-1} & 0 \\
0 & 0
\end{array}\right) \\
& M^{1}(X)=\left(\begin{array}{cc}
C X_{1,1} C+R & C X_{1,2} \\
C X_{1,2} & X_{2,2}
\end{array}\right)^{-1}
\end{aligned}
$$

A.2.3 Proof of Phase Transition Theorem. Here we use an adaptation of the proof by [27]. We first consider the two extremes of $\lambda=1$ and $\lambda=0$ and show they correspond to convergence and divergence. In the first case, $\lambda=1$, the modified algebraic Riccati equation (53) reduces to the standard algebraic Riccati equation, which converges to a fixed point, under the standard convergence hypotheses implied by Lemma (A.2). Thus, for any initial conditions $P_{0} \geq 0$, the covariance matrix remains bounded. In the second case, $\lambda=0$, the system reduces to open-loop prediction for the running sum $s_{t}$; and since the transition matrix $A$ is unstable, the covariance diverges for some initial $P_{0} \geq 0$.

It remains to show the existence of a single point of transition between the two cases. Fix a $0<\lambda_{1} \leq 1$ such that $\mathbb{E}_{\lambda_{1}}\left[P_{t+1}\right]$ is bounded for any initial condition $P_{0} \geq 0$. Then we show for any $\lambda_{2} \geq \lambda_{1}, \mathbb{E}_{\lambda_{2}}\left[P_{t}\right]$ is also bounded for all $P_{0} \geq 0$ :

$$
\begin{aligned}
\mathbb{E}_{\lambda_{1}}\left[P_{t+1}\right]= & \mathbb{E}_{\lambda_{1}}\left[\tilde{A} P_{t} \tilde{A}^{\prime}+\tilde{Q}\right. \\
& \left.-\tilde{A} P_{t} \tilde{C}\left[\left(1-\gamma_{t+1}\right) M^{0}\left(P_{t}\right)+\gamma_{t+1} M^{1}\left(P_{t}\right)\right] \tilde{C}^{\prime} P_{t} \tilde{A}^{\prime}\right] \\
= & \mathbb{E}\left[\tilde{A} P_{t} \tilde{A}^{\prime}+\tilde{Q}\right. \\
& \left.-\tilde{A} P_{t} \tilde{C}\left[\left(1-\lambda_{1}\right) M^{0}\left(P_{t}\right)+\lambda_{1} M^{1}\left(P_{t}\right)\right] \tilde{C}^{\prime} P_{t} \tilde{A}^{\prime}\right] \\
& \left(\text { by linearity of expectation, and } \gamma_{t+1} \Perp P_{t}\right) \\
= & \mathbb{E}\left[g_{\lambda_{1}}\left(P_{t}\right)\right] \\
\geq & \mathbb{E}\left[g_{\lambda_{2}}\left(P_{t}\right)\right] \text { by the Monotonicity Lemma } \\
= & \mathbb{E} \lambda_{\lambda_{2}}\left[P_{t+1}\right]
\end{aligned}
$$

So we can choose:

$$
\lambda_{c}=\left\{\inf \lambda^{*}: \lambda>\lambda^{*} \Longrightarrow \forall P_{0} \geq 0, \mathbb{E}_{\lambda}\left[P_{t}\right] \text { is bounded }\right\}
$$

Furthermore, by Lemma A.3, it follows that if $\lambda>0$, we can construct an estimator with bounded variance with probability one. And since our estimator is optimal, our estimator must have bounded variance. Thus $\lambda_{c}=0$ in the scalar case. Next we prove the vector case. 
A.2.4 Proof of Phase Transition Theorem (Vector Case). We argue that the Phase Transition Theorem holds in the vector case - for any $n$ (the dimension of both the latent state vectors $x_{t}$ and $s_{t}$ ), and for arbitrary $m$ (the dimension of $y_{t}$, the observation of $x_{t}$ ). We use proof by induction on $n$. The base case $n=1$ holds for any $m$ by Lemma A.3, since for any $\lambda>0$ we can construct a bounded estimator not dependent on $y_{t}$ or $m$. And the case of $\lambda=0$ results in unbounded estimation by the preceding argument - since $s_{t}$ is never observed.

For the inductive step, we consider the $(n+1)$-th dimension separately. If $\lambda>0$, then by Lemma A.3 we can construct an estimator for the $(n+1)$-th dimension with bounded error. And that estimator is not dependent on $y_{t}$ or $m$. Again, if $\lambda=0$, we have open-loop prediction for the $(n+1)$-th dimension, an unstable transition matrix, and thus unbounded covariance.

A.2.5 Proof of Monotonicity Theorem. From the Phase Transition Theorem, we know $\lambda_{c}=0$. Thus, both $\mathbb{E}_{\lambda_{1}}\left[P_{t}\right]$ and $\mathbb{E}_{\lambda_{2}}\left[P_{t}\right]$ are finite. Then the inequality follows from the Monotonicity Lemma.

\section{A.2.6 Lemmas.}

Lemma A.1 (Monotonicity). Assume $m=n=1$; i.e. $x_{t}, s_{t}, y_{t}, y_{t}^{s} \in \mathbb{R}$. Given $\lambda_{1}, \lambda_{2} \in \mathbb{R}$ with $\lambda_{1} \leq \lambda_{2}$; and $X$ a real PSD $2 \times 2$ matrix with $X_{2,2}>0$ then:

$$
g_{\lambda_{1}}(X) \geq g_{\lambda_{2}}(X)
$$

where the inequality indicates that the matrix difference is positive semidefinite.

Proof:

$$
g_{\lambda_{1}}(X)-g_{\lambda_{2}}(X)=\left(\lambda_{2}-\lambda_{1}\right)(\tilde{A} X \tilde{C})\left[M^{1}(X)-M^{0}(X)\right](\tilde{A} X \tilde{C})^{\prime}
$$

It remains to show that $M^{1}(X)-M^{0}(X)$ is PSD. By direct computation, we have:

$$
\begin{aligned}
& M^{1}(X)=\left(\begin{array}{cc}
X_{2,2} & -C X_{1,2} \\
-C X_{1,2} & C X_{1,1} C+R
\end{array}\right) * \frac{1}{X_{2,2}\left(C X_{1,1} C+R\right)-C^{2} X_{1,2}^{2}} \\
& \Longrightarrow M^{1}(X)-M^{0}(X)= \\
&\left(\begin{array}{cc}
X_{2,2}-\frac{X_{2,2}\left(C^{2} X_{1,1}+R\right)-C^{2} X_{1,2}^{2}}{C^{2} X_{1,1}+R} & -C X_{1,2} \\
-C X_{1,2} & C^{2} X_{1,1}+R
\end{array}\right) \\
& * \frac{1}{X_{2,2}\left(C^{2} X_{1,1}+R\right)-C^{2} X_{1,2}^{2}} \\
&=\left(\begin{array}{cc}
\frac{C^{2} X_{1,2}^{2}}{C^{2} X_{1,1}+R} & -C X_{1,2} \\
-C X_{1,2} & C^{2} X_{1,1}+R
\end{array}\right) * \frac{1}{X_{2,2}\left(C^{2} X_{1,1}+R\right)-C^{2} X_{1,2}^{2}}
\end{aligned}
$$

This last matrix has determinant zero; so at least one eigenvalue is zero. If we can show the trace is positive, then the other eigenvalue is positive and $M^{1}(X)-M^{0}(X)$ is PSD. 


$$
\begin{aligned}
\operatorname{trace}\left(M^{1}(X)-M^{0}(X)\right)= & \frac{C^{2} X_{1,2}^{2}+\left(C^{2} X_{1,1}+R\right)^{2}}{X_{2,2}-\frac{C^{2} X_{1,2}^{2}}{C^{2} X_{1,1}+R}} \\
& =\frac{>0}{\frac{\left|C^{2} X\right|+X_{2,2,}}{>0}}
\end{aligned}
$$

Which is positive, since by hypothesis $X$ is PSD, $X_{2,2}>0$ and $R>0$. The result follows.

Lemma A.2 (Control-Detect-Stability). A is unstable; $\left(\tilde{A}, \tilde{Q}^{1 / 2}\right)$ is controllable; and $(\tilde{A}, \tilde{C})$ is detectable.

Proof: We start with instability. First we note the $\tilde{A}=\left(\begin{array}{ll}A & 0 \\ A & 1\end{array}\right)$ has $\{A, 1\}$ as eigenvalues in the $2 \mathrm{x} 2$ case (where $A$ is scalar). Thus $\tilde{A}$ is unstable independent of $A$.

For controllable, note that we can write:

$$
\begin{aligned}
& \qquad \tilde{Q}=\sqrt{Q}\left(\begin{array}{l}
1 \\
1
\end{array}\right)\left(\begin{array}{ll}
1 & 1
\end{array}\right) \sqrt{Q}=\tilde{Q}^{1 / 2}\left(\tilde{Q}^{1 / 2}\right)^{\prime} \\
& \text { where } \tilde{Q}^{1 / 2}=\sqrt{Q}\left(\begin{array}{l}
1 \\
1
\end{array}\right)
\end{aligned}
$$

So the $2 x 2$ matrix $\left[\tilde{Q}^{1 / 2}, \tilde{A} \tilde{Q}^{1 / 2}\right]=\sqrt{Q}\left(\begin{array}{cc}1 & A \\ 1 & A+1\end{array}\right)$ has full rank, since its determinant is $\sqrt{Q}>0$. And thus by definition $\left(\tilde{A}, \tilde{Q}^{1 / 2}\right)$ is controllable.

Finally, we show pair $(\tilde{A}, \tilde{C})$ is detectable since. First note that $s_{t} \rightarrow 0 \Longrightarrow x_{t} \rightarrow 0$

$$
\tilde{C} X_{t}=\left(\begin{array}{c}
C x_{t} \\
s_{t}
\end{array}\right) \rightarrow 0 \Longleftrightarrow s_{t} \rightarrow 0
$$

where the last line implies $x_{t} \rightarrow 0$.

Lemma A.3 (Transition AT 0). If $\lambda>0$, then for any $t$, there exist estimators $\hat{x}_{t}, \hat{s}_{t}$ for $x_{t}, s_{t}$ with bounded variance with probability one.

Proof: Without loss of generality, assume we observe the sum $s_{t}$ at times $t_{2}$ and $t_{1}$ where $t_{2}>t_{1}$ with negligible additive noise (via $y_{t}^{s}$ ). We start by constructing an estimator $\hat{x}_{t_{1} \mid t_{2}}$ for $x_{t_{1}}$ given the observations up to time $t_{2}$. If $k>0$ we can write:

$$
\begin{aligned}
x_{t+k} & =A^{k} x_{t}+f\left(A, w_{t+1}^{t+k}\right) \\
\text { where } f\left(A, w_{t+1}^{t+k}\right) & =\sum_{i=1}^{k} A^{k-i} w_{t+i} \\
\text { and } \quad w_{t+1}^{t+k} & =\left(w_{t+1}, \ldots, w_{t+k}\right)
\end{aligned}
$$

Then:

$$
s_{t+k}-s_{t}=\sum_{i=1}^{t+k} x_{t+i}=x_{t} J(A, k)+\bar{f}\left(A, w_{t+1}^{t+k}\right)
$$

where $J(A, k)=\sum_{i=1}^{k} A^{i}$

and $\bar{f}\left(A, w_{t+1}^{t+k}\right)=\sum_{i=1}^{k} f\left(A, w_{t+1}^{t+i}\right)$ 
Since we observe $s_{t_{1}}$ and $s_{t_{2}}$, a valid estimator for $x_{t_{1} \mid t_{2}}$ is:

$$
\begin{aligned}
\hat{x}_{t_{1} \mid t_{2}} & =\frac{s_{t_{2}}-s_{t_{1}}}{J\left(A, t_{2}-t_{1}\right)} \\
\Longrightarrow\left(\hat{x}_{t_{1} \mid t_{2}}-x_{t_{1}}\right)^{2} & =\left[\frac{\bar{f}\left(A, w_{t_{1}+1}^{t_{2}}\right)}{J\left(A, t_{2}-t_{1}\right)}\right]^{2} \\
\Longrightarrow E\left(\hat{x}_{t_{1} \mid t_{2}}-x_{t_{1}}\right)^{2} & <\infty
\end{aligned}
$$

Where the last line follows since $t_{2}-t_{1}$ is finite, $J\left(A, t_{2}-t_{1}\right)$ is deterministic and finite, and $\bar{f}\left(A, w_{t_{1}+1}^{t_{2}}\right)$ is a non-central chi-square distribution with finite variance.

Then consider any $t_{3}>t_{2}$ such that no additional observations of $s_{t}$ have occured since time $t_{2}$. We can now use our estimator $\hat{x}_{t_{1} \mid t_{2}}$ to estimate $x_{t_{3}}$. Let $k=t_{3}-t_{1}$. Then:

$$
\begin{aligned}
x_{t_{3}} & =A^{k} x_{t_{1}}+f\left(A, w_{t_{1}+1}^{t_{3}}\right) \\
\text { Set } \quad \hat{x}_{t_{3}} & :=A^{k} \hat{x}_{t_{1} \mid t_{2}} \\
\Longrightarrow x_{t_{3}}-\hat{x}_{t_{3}} & =A^{k}\left(\hat{x}_{t_{1} \mid t_{2}}-x_{t_{1}}\right)+f\left(A, w_{t_{1}+1}^{t_{3}}\right) \\
\Longrightarrow E\left(x_{t_{3}}-\hat{x}_{t_{3}}\right)^{2} & =e(k)<\infty
\end{aligned}
$$

Where the last line follows, since the $w_{i}$ do not depend on $i$. Now since we assume $\lambda>0$, then with probability $1 k$ is finite. So our estimator $\hat{x}_{t_{3}}$ has bounded MSE. It follows that we can can estimate $\hat{s}_{t_{3}}$ with bounded MSE.

\section{A.3 System Identification \& Inference Proof}

Here we prove the End-to-End Convergence Theorem 3.8. Our approach is to establish that the state and covariance estimate Eqs. (30) - (34) are continuous functions of a.s. convergent random-variable sequences: functions of $\hat{\Theta}=(\hat{A}, \hat{C}, \hat{R}, \hat{Q})$. Then since $\hat{\Theta} \rightarrow \Theta$ with probability 1 , the result will follow. The Theorem is for case $m=n=1$; i.e. $x_{t}, s_{t}, y_{t}, y_{t}^{s} \in \mathbb{R}$, but generalization is straightforward.

Recall from Section 3.3 that $\hat{\Theta}=\hat{\Theta}(k)$, where $\mathrm{k}$ is the number of observations of the aggregate state $s_{t}$. We omit the dependence on $k$ when notationally convenient. We use the notation $\stackrel{a . s .}{\longrightarrow}$ to denote almost sure convergence with respect to the Frobenius norm.

We begin by showing that $M_{t+1 \mid t}^{0}(\hat{\Theta}) \stackrel{\text { a.s. }}{\longrightarrow} M_{t+1 \mid t}^{0}(\Theta)$ and $M_{t+1 \mid t}^{1}(\hat{\Theta}) \stackrel{\text { a.s. }}{\longrightarrow} M_{t+1 \mid t}^{1}(\Theta)$. Recall

$$
\begin{aligned}
& M_{t+1 \mid t}^{0}(\hat{\Theta}):=\left(\begin{array}{cc}
\left(\hat{C} P_{t+1 \mid t}^{x} \hat{C}+\hat{R}\right)^{-1} & 0 \\
0 & 0
\end{array}\right) \\
& M_{t+1 \mid t}^{1}(\hat{\Theta}):=\left(\begin{array}{cc}
\hat{C} P_{t+1 \mid t}^{x} \hat{C}+\hat{R} & \hat{C} P_{t+1 \mid t}^{x, s} \\
\hat{C} P_{t+1 \mid t}^{x, s} & P_{t+1 \mid t}^{s}
\end{array}\right)^{-1}
\end{aligned}
$$

Since $\left(C P_{t+1 \mid t}^{x} C+R\right)$ is non-zero, if $k \gg 1,\left(\hat{C} P_{t+1 \mid t}^{x} \hat{C}+\hat{R}\right)$ is bounded away from zero almost surely. Then since the one-dimensional multiplicative inverse is continuous except at zero, $\left(\hat{C} P_{t+1 \mid t}^{x} \hat{C}+\right.$ $\hat{R})^{-1} \stackrel{\text { a.s. }}{\longrightarrow}\left(C P_{t+1 \mid t}^{x} C+R\right)^{-1}$. That is, $M_{t+1 \mid t}^{0}(\hat{\Theta}) \stackrel{\text { a.s. }}{\longrightarrow} M_{t+1 \mid t}^{0}(\Theta)$. 
Next, by continuity of matrix multiplication and addition, and by the continuous mapping theorem

$$
\left(\begin{array}{cc}
\hat{C} P_{t+1 \mid t}^{x} \hat{C}+\hat{R} & \hat{C} P_{t+1 \mid t}^{x, s} \\
\hat{C} P_{t+1 \mid t}^{x, s} & P_{t+1 \mid t}^{s}
\end{array}\right) \stackrel{\text { a.s. }}{\longrightarrow}\left(\begin{array}{cc}
C P_{t+1 \mid t}^{x} C+R & C P_{t+1 \mid t}^{x, s} \\
C P_{t+1 \mid t}^{x, s} & P_{t+1 \mid t}^{s}
\end{array}\right)
$$

Next we note that the determinant operator and adjunct for an invertible $2 \times 2$ matrix are continuous functions; so by the continuous mapping theorem

$$
\left(\begin{array}{cc}
\hat{C} P_{t+1 \mid t}^{x} \hat{C}+\hat{R} & \hat{C} P_{t+1 \mid t}^{x, s} \\
\hat{C} P_{t+1 \mid t}^{x, s} & P_{t+1 \mid t}^{s}
\end{array}\right)^{-1} \stackrel{\text { a.s. }}{\longrightarrow} M_{t+1 \mid t}^{1}(\Theta)
$$

Continuing, from equation 29 we see that trivially

$$
\tilde{A}(\hat{\Theta}) \stackrel{\text { a.s. }}{\longrightarrow} \tilde{A}(\Theta) \quad \tilde{C}(\hat{\Theta}) \stackrel{\text { a.s. }}{\longrightarrow} \tilde{C}(\Theta) \quad \tilde{Q}(\hat{\Theta}) \stackrel{\text { a.s. }}{\longrightarrow} \tilde{Q}(\Theta)
$$

Finally, we note that each random variable in the right-hand side of Eqs. (30)-(34) is either given, fixed, one of the convergent sequence discussed above (Eqs. 27, 28, 29), or a trivially continuous function of $\hat{\Theta}$. Furthermore, Eqs. (30)-(34) consist of matrix multiplication, addition, and transpose, and so are trivially continuous functions of their arguments. The result follows by the continuous mapping theorem.

Received August 2019; revised September 2019; accepted October 2019 